\title{
Inequalities in Delayed Initiation of Breastfeeding: Evidence from Health Surveys in 58 Low- and Middle-Income Countries (2012-2017)
}

Shahreen Raihana ( $\nabla$ srai7309@uni.sydney.edu.au )

The University of Sydney https://orcid.org/0000-0003-1285-8867

Michael J Dibley

The University of Sydney School of Public Health

Sabrina Sharmin Priyanka

ICDDRB: International Centre for Diarrhoeal Disease Research Bangladesh

Ashraful Alam

The University of Sydney School of Public Health

Tanvir M Huda

The University of Sydney School of Public Health

\section{Research}

Keywords: Breastfeeding, initiation, delayed, inequality, wealth, socio-economic, low- and middle-income countries

Posted Date: September 27th, 2021

DOI: https://doi.org/10.21203/rs.3.rs-923831/v1

License: (c) (i) This work is licensed under a Creative Commons Attribution 4.0 International License. Read Full License 


\section{Abstract}

Background: Delay in breastfeeding initiation beyond 1 hour of birth increases the risk of neonatal deaths and illnesses. Despite ample evidence highlighting its importance, wealth-related inequalities exist in delayed breastfeeding initiation rates in many low-and middle-income countries (LMICs). Our goal was to examine the magnitude and trend of socio-economic inequalities in delayed breastfeeding initiation rates in LMICs.

Methods: We used data from 58 low- and middle-income countries from their most recent Demographic and Health Survey and Multiple Indicator Cluster Survey to present delayed breastfeeding initiation rates across wealth quintile and area of residence. To assess the wealth-related inequalities in delayed breastfeeding initiation rates, we calculated two indicators- the difference, in percentage points, between the rates recorded for the poorest and wealthiest quintiles and the ratio of the same two values. To present the change over time, we calculated- the annual absolute change and annual absolute excess change.

Results: Within countries, the prevalence of delayed breastfeeding initiation was higher among the wealthiest quintile (median $47.4 \%$ ) and lower in the poorest quintile (median 42.6\%). In 37 study countries, the prevalence of delayed breastfeeding initiation was higher among the wealthiest than among the poorest. The highest prevalence of delayed breastfeeding initiation was in South Asia, and the largest levels of pro-rich inequality were in Latin America and Caribbean. Pro-poor inequality was more common in Sub-Saharan Africa. Delayed breastfeeding initiation rates decreased faster in the poorest quintile (median -1.3 percent-points per year) compared to the wealthiest quintile (median -0.8 percent-points per year), indicating an increase in pro-poor inequality over time.

Conclusions: There is no distinguishable global pattern for wealth-related inequality in the prevalence of delayed breastfeeding initiation. The prevailing inequality may be due to a combination of sociodemographic factors and is largely preventable by incorporating adequate infrastructure towards creating a pro-breastfeeding environment.

\section{Introduction}

The World Health Organization (WHO) recommends that all infants be put to their mother's breast immediately after birth to ensure early optimum growth and development of newborns is sustained through infancy [1]. In addition, global guidelines, including the Baby-friendly hospital initiative (BFHI) [2] and early essential newborn care (EENC) [3], outline the importance of initiation of breastfeeding within the first hour of birth. Initiation of breastfeeding within 1 hour of birth is necessary because it helps enhance a newborn's immune response to infectious pathogens $[4,5]$ and protects them from infection associated newborn deaths [6,7] and severe illnesses [8]. Furthermore, initiating breastfeeding on time has a limited direct cost and is highly cost-effective in preventing adverse health outcomes extending throughout infancy [9]. Systematic reviews [7, 10], pooled analysis [11], primary studies [12, 13], and global reports by UNICEF and WHO $[14,15]$ all confirm that delaying breastfeeding initiation beyond 1 hour of birth is associated with at least 1.3 times higher risk newborn deaths. The risk of newborn deaths further increases to 2.4 times higher risk when breastfeeding is initiated after the first day [10].

Despite strong evidence supporting breastfeeding initiation within 1 hour of birth, three-fifths of the world's newborns ranging from as high as $68 \%$ of newborns in East Asia and Pacific to 35\% in Eastern and Southern Africa, begin breastfeeding beyond the first hour [14, 16]. According to the WHO's tool for measuring and rating infant and young child feeding practices, the global prevalence of delayed breastfeeding initiation falls considerably short of a 'very good' score [17] with four of the WHO regions (East Asia and Pacific, Middle East and North Africa, South Asia and Western and Central Africa) performing at a 'fair' rate.

Equity in maternal and child health indicators implies that all women and children can access their 'full health potential' and that socio-economic status does not cause anyone to be left behind [18]. Inequality in breastfeeding indicators has not been static over the years. While there is no prevailing pattern in delayed breastfeeding initiation globally $[14,19]$, studies in many countries have reported socio-economic factors relating to wealth associated with delayed breastfeeding initiation [20-27], where wealthier women are often delaying breastfeeding initiation beyond the first hour of birth.

The global strategy for Every Woman Every Child identifies breastfeeding initiation within 1 hour of birth and exclusive breastfeeding for six months as crucial towards achieving SDG targets on child survival, health and nutrition [28]. While there is ample evidence of socio-economic inequalities in continued breastfeeding till 23 months of child's age [16], there is limited evidence on the inequalities in delayed breastfeeding initiation and exclusive breastfeeding till six months [29]. An independent expert review group on information and accountability for women's and children's health identifies breastfeeding as a maternal or child health indicator that displays a pro-poor nature [30]. The worldwide prevalence of delayed breastfeeding initiation decreased from $68 \%$ in 2000 to $51 \%$ in 2020 [15]. But unlike other breastfeeding indicators, there has been no multi-country exploration to confirm the nature of the inequality in this crucial indicator. Evaluating progress only at the global level does not account for critical socio-economic factors contributing to pro-poor or pro-rich patterns across and within countries. Measuring and monitoring inequalities in delayed breastfeeding initiation across countries can help identify higher-risk subgroups and inform the design of equity-oriented interventions to support timely breastfeeding initiation as appropriate for the country.

This paper explores the economic inequalities in the prevalence of delayed breastfeeding initiation in 58 low- and middle-income countries spanning all world regions and presents how the prevalence changed over ten years. We report how women at the extreme ends of the wealth index within these countries are at disadvantageous positions related to the initiation of breastfeeding.

\section{Methods}


We obtained the data for this study from 58 publicly available nationally representative cross-sectional surveys conducted in low-and middle-income countries (LMICs). Data were collected from Demographic and Health Surveys (DHS) of 49 countries and Multiple Indicator Cluster Surveys (MICS) [31, 32] of 9 countries conducted between 2012 and 2017 and reported before January 2020. We selected the latest survey available for these 58 low- and middleincome countries during the period and used data on household assets required to construct a standard household wealth index and breastfeeding status of children born in the two years preceding the survey. For countries with no DHS survey data between 2012 and 2017, we looked for a MICS survey conducted during the same period and, if available, included it in this study.

The DHS and MICS are reasonably comparable in their sampling methods, questionnaires, indicators, and data collection procedures [33]. These surveys report comparative information on sociodemographic characteristics, household assets and maternal and child health and nutrition. Both DHS and MICS surveys employed a multistage sampling procedure to collect individual and household-level data from face-to-face interviews of women aged 15-49 years. They both used standardized questionnaires administered by trained interviewers and standardized measurement tools across time and countries to ensure uniformity of the reported indicators. Both surveys are typically administered, conducted and implemented by national statistical agencies of respective countries. Further, both surveys use sample weights to ensure the sample is representative of the reference population.

\section{Study Sample}

The study sample comprised all women who had a live birth in the two years preceding the survey. We extracted data from the 'birth' and 'household' record files for DHS and the 'women', 'household' and 'child' record files for MICS. We did not include eight surveys conducted during the same period in this analysis. Among them, Colombia DHS 2015 survey did not collect data or report the time of initiation of breastfeeding. We could not convert the labelled datasets for Congo and El Salvador MICS 2014 surveys to Stata format. The weighted numbers of women whose last child was born in the two years preceding the survey presented in the Kenya DHS 2014 and Turkey DHS 2013 did not match the weighted numbers calculated by the author. The Paraguay MICS 2016 survey did not include the sample weights in the publicly available dataset. The Mexico MICS 2015 and Peru DHS 2014 did not include unique identifiers in the birth and household files, preventing file merging.

Data were cleaned and restricted to women who had a live birth in the 24 months preceding the survey. Variables in the restricted data files for each country were then uniformly labelled and appended into one data file comprising relevant variables for all 58 countries. According to the World Bank regions, we added new variables to group the countries [34]. The six World Bank regions considered are-i) East Asia \& Pacific, ii) Europe \& Central Asia, iii) Latin America \& Caribbean, iv) the Middle East \& North Africa, v) South Asia, vi) Sub-Saharan Africa [34]. Supplementary Table 1 presents information on each survey, including the DHS country code, survey year, region, number of women interviewed, and the number of households covered.

\section{Measures}

'Delayed breastfeeding initiation' is the primary outcome of interest in this study. We defined delayed initiation as women who reported having initiated breastfeeding after the first hour of birth. It included children born alive in the two years preceding the surveys and put to their mother's breast one hour or more after birth. The survey interviewers asked women aged 15-49 with a live birth in the two years preceding the survey when they put their child to the breast using the following unprompted self-reported question- 'How long after birth did you first put (name of child) to the breast?'. We used the WHO recommended cut off for timely initiation of breastfeeding [1].

None of the DHS and MICS surveys collected data on household income and expenditure. Thus, we used the household wealth index as a proxy indicator to determine household socio-economic status to ascertain rich-poor differentials $[35,36]$. We used principal component analysis to construct a wealth index based on ownership of selected household assets, housing quality, water and sanitation facilities, and place of residence [35]. We used 12 common household assets and housing materials to compute the household wealth index [37-39]. Each household was thus assigned a wealth score representing its position relative to other households within the country. We divided these scores into quintiles, with the first quintile (Q1) representing the poorest 20\% of households and the fifth quintile (Q5) representing the wealthiest $20 \%$. To describe the change in inequality over the preceding decade, we extracted additional information on the historical prevalence of delayed breastfeeding initiation from countries that reported it between 2002 and 2007.

\section{Statistical Analysis}

We presented the prevalence of delayed breastfeeding initiation at the national level and for each wealth quintile. We examined the absolute socioeconomic inequality in delayed breastfeeding initiation by subtracting the prevalence among the poorest from the wealthiest. In addition, we used the ratio of the prevalence in the wealthiest and poorest as a measure of relative inequality. We also demonstrated the correlation between a country's income inequality and absolute wealth-related inequality in delayed initiation of breastfeeding. We used the Gini coefficient as a relative measure of a country's income inequality with values ranging from 0 (complete equality) to 1 (perfect inequality) [40,41]. The Gini index assumes redistribution of income across the country's residents by raising the income of the poorest at the cost of a reduced income of the wealthiest [40]. We extracted the Gini index of each country from the World Bank database [41] if a Gini index was available for each included country in the survey year or within five years before the survey. This ensured time appropriate representation of the country's level of inequality around a similar time to the survey. Gini index was not included in this analysis if none were reported five years before the survey. We further present the prevalence of delayed breastfeeding initiation across wealth quintiles, stratified by place of residence.

To report the change in pace over an approximately 10-year period, we calculated the absolute difference in the prevalence of delayed breastfeeding initiation between 2 survey years of the same country. First, we took the absolute change in the national prevalence and then reported change over time within the poorest and wealthiest quintiles. Then, to compare the rate of change in prevalence between the two quintiles per year, we calculated the annual

Page $3 / 20$ 
absolute excess change by subtracting the absolute difference in the wealthiest from the absolute change in the poorest and then dividing it by the number of years between the two surveys [19]. Considering the health outcome we are exploring is an 'undesirable' one [19], a positive excess change indicates that the rate of change was more favourable for the wealthiest than the rate of change in the poorest quintile. Similarly, a negative value indicates that the rate of change in prevalence was more favourable for the poorest group.

We performed all statistical analyses using STATA version 15 (Stata Corporation, College Station, TX). Statistical significance was considered at a 95\% confidence interval for prevalence estimates and absolute and relative inequality measures. We used the sample weights already in the datasets to obtain all country-level estimates. We then used the number of women 15-49 years in each of these countries as reported by the United Nations Development Programme (UNDP) population survey to de-normalize the standard weights to ensure the appropriate contribution of data within each country [42]. The analysis settings of the dataset were assigned using the command 'svyset' in Stata to designate the sampling unit, survey weights and the cluster design of the survey and obtain standard errors of all estimates.

\section{Results}

Our analysis included a total of 298,656 women from 58 countries who had a live birth in the two years preceding each survey. In the appended dataset of all countries, about $53.8 \%$ had breastfeeding initiated after the first hour of birth.

Table 1 (and Supplementary Fig. 1) presents the delayed breastfeeding initiation rates across all wealth quintiles and the absolute (difference) and relative (ratio) inequality in the prevalence between the wealthiest and the poorest quintiles. The prevalence of delayed initiation of breastfeeding ranged from $15.0 \%$ in Burundi to $83.4 \%$ in Guinea. In more than one third $(n=21)$ of the study countries breastfeeding initiation rates were higher than $50 \%$, which has been rated by WHO as "fair" performance based on past analysis of the prevalence of timely breastfeeding initiation in developing countries [17]. According to the same WHO tool, none of the included countries had a prevalence of delayed breastfeeding initiation lower than $10 \%$ - cut off for "very good" performance [17]. In 25 study countries, the delayed initiation was higher than $50 \%$ ("fair") in the wealthiest quintile, including eight countries with a prevalence of more than $70 \%$ ("poor") among the wealthiest. In comparison, the prevalence in 18 countries in the poorest quintile was higher than $50 \%$, five of which had a prevalence greater than $70 \%$.

Table 1: Delayed breastfeeding initiation rates by economic status in 58 low- and middle-income countries, by World Bank region (demographic and health surveys and multiple indicator cluster surveys 2012-2017). Values are percentages (95\% confidence intervals) 


\begin{tabular}{|c|c|c|c|c|c|c|c|c|}
\hline \multirow[t]{2}{*}{ Region/Country } & \multirow{2}{*}{$\begin{array}{l}\text { National prevalence of } \\
\text { delayed initiation of } \\
\text { breastfeeding }\end{array}$} & \multicolumn{5}{|c|}{ Prevalence of delayed initiation across wealth quintiles } & \multirow{2}{*}{$\begin{array}{l}\text { Difference in } \\
\text { percentage } \\
\text { points ( } 5 \text { th } \\
\text { fifth }-1 \text { st fifth) }\end{array}$} & \multirow{2}{*}{$\begin{array}{l}\text { Ratio } \\
\text { (5th } \\
\text { fifth:1st } \\
\text { fifth) }\end{array}$} \\
\hline & & $\begin{array}{l}\text { 1st fifth } \\
\text { (poorest) }\end{array}$ & 2nd fifth & 3rd fifth & 4th fifth & $\begin{array}{l}\text { 5th fifth } \\
\text { (wealthiest) }\end{array}$ & & \\
\hline \multicolumn{9}{|l|}{$\begin{array}{l}\text { East Asia \& } \\
\text { Pacific }\end{array}$} \\
\hline Thailand & $60.1[55.6,64.4]$ & $\begin{array}{l}47.0 \\
{[38.6,55.6]}\end{array}$ & $\begin{array}{l}56.9 \\
{[49.1,64.3]}\end{array}$ & $\begin{array}{l}58.3 \\
{[49.2,66.8]}\end{array}$ & $\begin{array}{l}64.0 \\
{[55.0,72.0]}\end{array}$ & $\begin{array}{l}72.8 \\
{[61.7,81.6]}\end{array}$ & $\begin{array}{l}25.7 \\
{[12.5,39.0]}\end{array}$ & $\begin{array}{l}1.5 \\
{[1.2,1.9]}\end{array}$ \\
\hline Indonesia & $43.4[41.7,45.1]$ & $\begin{array}{l}45.3 \\
{[42.4,48.2]}\end{array}$ & $\begin{array}{l}40.1 \\
{[36.9,43.5]}\end{array}$ & $\begin{array}{l}47.2 \\
{[43.8,50.6]}\end{array}$ & $\begin{array}{l}41.1 \\
{[37.8,44.5]}\end{array}$ & $\begin{array}{l}43.5 \\
{[39.4,47.6]}\end{array}$ & $-1.8[-6.8,3.2]$ & $\begin{array}{l}1.0 \\
{[0.8,1.1]}\end{array}$ \\
\hline Philippines & $43.1[40.4,45.9]$ & $\begin{array}{l}38.3 \\
{[33.9,43.0]}\end{array}$ & $\begin{array}{l}39.4 \\
{[35.0,44.0]}\end{array}$ & $\begin{array}{l}42.2 \\
{[36.7,48.0]}\end{array}$ & $\begin{array}{l}44.8 \\
{[39.1,50.7]}\end{array}$ & $\begin{array}{l}50.6 \\
{[43.4,57.8]}\end{array}$ & $12.3[3.8,20.8]$ & $\begin{array}{l}1.3 \\
{[1.1,1.6]}\end{array}$ \\
\hline Cambodia & $37.4[34.8,40.2]$ & $\begin{array}{l}36.6 \\
{[31.6,41.8]}\end{array}$ & $\begin{array}{l}38.8 \\
{[33.6,44.2]}\end{array}$ & $\begin{array}{l}33.9 \\
{[29.1,39.0]}\end{array}$ & $\begin{array}{l}30.9 \\
{[25.5,36.8]}\end{array}$ & $\begin{array}{l}47.1 \\
{[41.0,53.2]}\end{array}$ & $10.5[2.5,18.6]$ & $\begin{array}{l}1.3 \\
{[1.0,1.5]}\end{array}$ \\
\hline Myanmar & $33.2[30.1,36.5]$ & $\begin{array}{l}35.2 \\
{[28.6,42.5]}\end{array}$ & $\begin{array}{l}36.9 \\
{[30.1,44.3]}\end{array}$ & $\begin{array}{l}32.3 \\
{[26.1,39.2]}\end{array}$ & $\begin{array}{l}30.8 \\
{[25.6,36.5]}\end{array}$ & $\begin{array}{l}30.9 \\
{[25.2,37.3]}\end{array}$ & $-4.3[-13.4,4.9]$ & $\begin{array}{l}0.9 \\
{[0.6,1.1]}\end{array}$ \\
\hline Timor Leste & $24.8[22.2,27.5]$ & $\begin{array}{l}29.8 \\
{[24.3,35.9]}\end{array}$ & $\begin{array}{l}21.1 \\
{[17.3,25.5]}\end{array}$ & $\begin{array}{l}24.3 \\
{[20.0,29.2]}\end{array}$ & $\begin{array}{l}24.7 \\
{[20.0,30.1]}\end{array}$ & $\begin{array}{l}23.9 \\
{[19.6,28.7]}\end{array}$ & $-5.9[-13.3,1.5]$ & $\begin{array}{l}0.8 \\
{[0.6,1.0]}\end{array}$ \\
\hline \multicolumn{9}{|l|}{$\begin{array}{l}\text { Europe \& } \\
\text { Central Asia }\end{array}$} \\
\hline Armenia & $59.1[54.1,63.9]$ & $\begin{array}{l}61.7 \\
{[49.1,72.9]}\end{array}$ & $\begin{array}{l}61.0 \\
{[52.5,68.9]}\end{array}$ & $\begin{array}{l}57 \\
{[46.6,66.9]}\end{array}$ & $\begin{array}{l}56.9 \\
{[45.4,67.8]}\end{array}$ & $\begin{array}{l}58.9 \\
{[49.5,67.7]}\end{array}$ & $\begin{array}{l}-2.8 \\
{[-18.0,12.4]}\end{array}$ & $\begin{array}{l}0.9 \\
{[0.7,1.2]}\end{array}$ \\
\hline Albania & $43.5[38.7,48.4]$ & $\begin{array}{l}35.6 \\
{[25.8,46.8]}\end{array}$ & $\begin{array}{l}33.4 \\
{[24.1,44.1]}\end{array}$ & $\begin{array}{l}54.0 \\
{[44.4,63.4]}\end{array}$ & $\begin{array}{l}49.6 \\
{[41.8,57.3]}\end{array}$ & $\begin{array}{l}44.8 \\
{[35.6,54.3]}\end{array}$ & $9.1[-5.0,23.3]$ & $\begin{array}{l}1.3 \\
{[0.8,1.7]}\end{array}$ \\
\hline Moldova & $39.1[35.0,43.3]$ & $\begin{array}{l}43.6 \\
{[32.3,55.5]}\end{array}$ & $\begin{array}{l}30.8 \\
{[23.0,40.0]}\end{array}$ & $\begin{array}{l}40.2 \\
{[30.9,50.2]}\end{array}$ & $\begin{array}{l}44.4 \\
{[35.4,53.7]}\end{array}$ & $\begin{array}{l}38.6 \\
{[32.3,45.3]}\end{array}$ & $-5.0[-18.7,8.7]$ & $\begin{array}{l}0.9 \\
{[0.6,1.2]}\end{array}$ \\
\hline Tajikistan & $38.4[35.0,41.9]$ & $\begin{array}{l}32.8 \\
{[28.3,37.7]}\end{array}$ & $\begin{array}{l}35.0 \\
{[29.5,40.9]}\end{array}$ & $\begin{array}{l}41.7 \\
{[35.7,47.9]}\end{array}$ & $\begin{array}{l}40.7 \\
{[35.0,46.7]}\end{array}$ & $\begin{array}{l}41.7 \\
{[33.2,50.8]}\end{array}$ & $8.9[-1.1,18.9]$ & $\begin{array}{l}1.3 \\
{[0.9,1.6]}\end{array}$ \\
\hline Ukraine & $34.3[30.5,38.4]$ & $\begin{array}{l}37.9 \\
{[30.1,46.4]}\end{array}$ & $\begin{array}{l}40.1 \\
{[33.2,47.5]}\end{array}$ & $\begin{array}{l}32.2 \\
{[24.6,40.8]}\end{array}$ & $\begin{array}{l}35.2 \\
{[26.4,45.1]}\end{array}$ & $\begin{array}{l}26.5 \\
{[19.9,34.5]}\end{array}$ & $\begin{array}{l}-11.4 \\
{[-22.4,-0.4]}\end{array}$ & $\begin{array}{l}0.7 \\
{[0.4,0.9]}\end{array}$ \\
\hline Kazakhstan & $16.7[14.7,18.9]$ & $\begin{array}{l}14.3 \\
{[10.4,19.3]}\end{array}$ & $\begin{array}{l}15.4 \\
{[10.4,22.2]}\end{array}$ & $\begin{array}{l}18.5 \\
{[13.5,25.0]}\end{array}$ & $\begin{array}{l}20.4 \\
{[16.3,25.3]}\end{array}$ & $\begin{array}{l}14.3 \\
{[10.3,19.6]}\end{array}$ & $0.1[-6.4,6.5]$ & $\begin{array}{l}1.0 \\
{[0.5,1.5]}\end{array}$ \\
\hline Kyrgyz Republic & $16.2[14.0,18.6]$ & $\begin{array}{l}13.2 \\
{[8.8,19.2]}\end{array}$ & $\begin{array}{l}16.3 \\
{[12.6,20.7]}\end{array}$ & $\begin{array}{l}13.6 \\
{[9.8,18.7]}\end{array}$ & $\begin{array}{l}15.4 \\
{[11.4,20.5]}\end{array}$ & $\begin{array}{l}22.4 \\
{[17.3,28.6]}\end{array}$ & $9.3[1.7,16.8]$ & $\begin{array}{l}1.7 \\
{[0.9,2.5]}\end{array}$ \\
\hline \multicolumn{9}{|l|}{$\begin{array}{l}\text { Latin America } \\
\text { and the } \\
\text { Caribbean }\end{array}$} \\
\hline $\begin{array}{l}\text { Dominican } \\
\text { Republic }\end{array}$ & $56.8[52.5,61.1]$ & $\begin{array}{l}48.3 \\
{[42.6,54.0]}\end{array}$ & $\begin{array}{l}55.1 \\
{[47.1,62.8]}\end{array}$ & $\begin{array}{l}58.4 \\
{[50.4,66.0]}\end{array}$ & $\begin{array}{l}59.2 \\
{[50.5,67.4]}\end{array}$ & $\begin{array}{l}63.3 \\
{[53.1,72.4]}\end{array}$ & $15.0[3.9,26.1]$ & $\begin{array}{l}1.3 \\
{[1.1,1.6]}\end{array}$ \\
\hline Haiti & $52.6[50.1,55.2]$ & $\begin{array}{l}50.5 \\
{[45.8,55.3]}\end{array}$ & $\begin{array}{l}49.0 \\
{[43.9,54.2]}\end{array}$ & $\begin{array}{l}51.7 \\
{[45.9,57.5]}\end{array}$ & $\begin{array}{l}52.2 \\
{[45.6,58.7]}\end{array}$ & $\begin{array}{l}59.7 \\
{[54.4,64.9]}\end{array}$ & $9.2[2.1,16.2]$ & $\begin{array}{l}1.2 \\
{[1.0,1.3]}\end{array}$ \\
\hline Guyana & $50.8[47.0,54.6]$ & $\begin{array}{l}42.2 \\
{[36.3,48.4]}\end{array}$ & $\begin{array}{l}41.3 \\
{[33.5,49.5]}\end{array}$ & $\begin{array}{l}58.0 \\
{[49.6,65.9]}\end{array}$ & $\begin{array}{l}52.5 \\
{[43.7,61.2]}\end{array}$ & $\begin{array}{l}72.0 \\
{[63.5,79.1]}\end{array}$ & $\begin{array}{l}29.7 \\
{[19.8,39.6]}\end{array}$ & $\begin{array}{l}1.7 \\
{[1.4,2.0]}\end{array}$ \\
\hline Guatemala & $36.9[34.9,38.9]$ & $\begin{array}{l}24.9 \\
{[21.7,28.3]}\end{array}$ & $\begin{array}{l}28.7 \\
{[25.5,32.2]}\end{array}$ & $\begin{array}{l}36.2 \\
{[32.3,40.1]}\end{array}$ & $\begin{array}{l}43.1 \\
{[38.9,47.4]}\end{array}$ & $\begin{array}{l}51.5 \\
{[47.2,55.8]}\end{array}$ & $\begin{array}{l}26.6 \\
{[21.2,32.1]}\end{array}$ & $\begin{array}{l}2.1 \\
{[1.7,2.4]}\end{array}$ \\
\hline \multicolumn{9}{|l|}{$\begin{array}{l}\text { The Middle } \\
\text { East \& North } \\
\text { Africa }\end{array}$} \\
\hline Egypt & $72.9[71.2,74.5]$ & $\begin{array}{l}70.4 \\
{[67.3,73.4]}\end{array}$ & $\begin{array}{l}71.9 \\
{[68.6,75.0]}\end{array}$ & $\begin{array}{l}73.7 \\
{[70.1,77.0]}\end{array}$ & $\begin{array}{l}74.0 \\
{[70.6,77.2]}\end{array}$ & $\begin{array}{l}74.5 \\
{[70.8,77.9]}\end{array}$ & $4.1[-0.6,8.7]$ & $\begin{array}{l}1.1 \\
{[1.0,1.1]}\end{array}$ \\
\hline Tunisia & $60.1[56.2,63.8]$ & $\begin{array}{l}54.5 \\
{[47.6,61.2]}\end{array}$ & $\begin{array}{l}54.7 \\
{[46.3,62.8]}\end{array}$ & $\begin{array}{l}61.4 \\
{[53.7,68.5]}\end{array}$ & $\begin{array}{l}62.6 \\
{[55.1,69.6]}\end{array}$ & $\begin{array}{l}67.9 \\
{[57.9,76.5]}\end{array}$ & $13.5[1.7,25.3]$ & $\begin{array}{l}1.2 \\
{[1.0,1.5]}\end{array}$ \\
\hline Yemen & $47.3[45.2,49.5]$ & $\begin{array}{l}45.3 \\
{[40.9,49.9]}\end{array}$ & $\begin{array}{l}48.8 \\
{[45.1,52.5]}\end{array}$ & $\begin{array}{l}46.5 \\
{[42.6,50.5]}\end{array}$ & $\begin{array}{l}47.6 \\
{[42.9,52.3]}\end{array}$ & $\begin{array}{l}48.3 \\
{[44.0,52.7]}\end{array}$ & $3.0[-3.3,9.3]$ & $\begin{array}{l}1.1 \\
{[0.9,1.2]}\end{array}$ \\
\hline Jordan & $33.0[30.4,35.6]$ & $\begin{array}{l}30.2 \\
{[23.8,37.4]}\end{array}$ & $\begin{array}{l}33.1 \\
{[28.0,38.7]}\end{array}$ & $\begin{array}{l}31.3 \\
{[26.6,36.4]}\end{array}$ & $\begin{array}{l}31.8 \\
{[26.9,37.1]}\end{array}$ & $\begin{array}{l}38.4 \\
{[34.3,42.6]}\end{array}$ & $8.2[0.3,16.1]$ & $\begin{array}{l}1.3 \\
{[1.0,1.6]}\end{array}$ \\
\hline \multicolumn{9}{|l|}{ South Asia } \\
\hline Pakistan & $80.4[77.9,82.7]$ & 81.3 & 77.4 & 79.7 & 80.4 & 83.4 & $2.1[-4.7,8.9]$ & 1.0 \\
\hline
\end{tabular}




\begin{tabular}{|c|c|c|c|c|c|c|c|c|}
\hline & & {$[74.9,86.4]$} & {$[72.8,81.4]$} & [75.5,83.3] & {$[75.7,84.4]$} & {$[79.3,86.9]$} & & {$[0.9,1.1]$} \\
\hline Afghanistan & $59.1[56.6,61.6]$ & $\begin{array}{l}57.0 \\
{[53.3,60.6]}\end{array}$ & $\begin{array}{l}56.4 \\
{[52.7,60.0]}\end{array}$ & $\begin{array}{l}62.9 \\
{[56.5,68.9]}\end{array}$ & $\begin{array}{l}59.6 \\
{[54.7,64.3]}\end{array}$ & $\begin{array}{l}59.7 \\
{[55.8,63.3]}\end{array}$ & $2.7[-2.6,7.9]$ & $\begin{array}{l}1.0 \\
{[0.9,1.1]}\end{array}$ \\
\hline India & $58.5[58.0,59.0]$ & $\begin{array}{l}59.4 \\
{[58.4,60.4]}\end{array}$ & $\begin{array}{l}59.4 \\
{[58.4,60.4]}\end{array}$ & $\begin{array}{l}58.3 \\
{[57.3,59.4]}\end{array}$ & $\begin{array}{l}58.3 \\
{[57.2,59.4]}\end{array}$ & $\begin{array}{l}57.1 \\
{[56.0,58.2]}\end{array}$ & $-2.3[-3.9,-0.7]$ & $\begin{array}{l}1.0 \\
{[0.9,1.0]}\end{array}$ \\
\hline Bangladesh & $49.2[46.5,51.9]$ & $\begin{array}{l}45.3 \\
{[39.8,51.0]}\end{array}$ & $\begin{array}{l}45.4 \\
{[39.5,51.5]}\end{array}$ & $\begin{array}{l}48.7 \\
{[42.4,55.0]}\end{array}$ & $\begin{array}{l}52.6 \\
{[47.3,57.8]}\end{array}$ & $\begin{array}{l}54.0 \\
{[49.3,58.6]}\end{array}$ & $8.7[1.5,15.9]$ & $\begin{array}{l}1.2 \\
{[1.0,1.4]}\end{array}$ \\
\hline Nepal & $45.1[42.0,48.1]$ & $\begin{array}{l}36.8 \\
{[31.7,42.1]}\end{array}$ & $\begin{array}{l}45.4 \\
{[40.2,50.7]}\end{array}$ & $\begin{array}{l}48.4 \\
{[42.3,54.7]}\end{array}$ & $\begin{array}{l}46.1 \\
{[39.2,53.1]}\end{array}$ & $\begin{array}{l}48.7 \\
{[42.9,54.6]}\end{array}$ & $12.0[4.2,19.7]$ & $\begin{array}{l}1.3 \\
{[1.1,1.6]}\end{array}$ \\
\hline Maldives & $33.5[29.5,37.8]$ & $\begin{array}{l}38.1 \\
{[30.2,46.7]}\end{array}$ & $\begin{array}{l}32.4 \\
{[23.3,43.2]}\end{array}$ & $\begin{array}{l}31.4 \\
{[21.9,42.8]}\end{array}$ & $\begin{array}{l}39.7 \\
{[31.2,49.0]}\end{array}$ & $\begin{array}{l}25.9 \\
{[20.1,32.7]}\end{array}$ & $\begin{array}{l}-12.2 \\
{[-22.6,-1.8]}\end{array}$ & $\begin{array}{l}0.7 \\
{[0.5,0.9]}\end{array}$ \\
\hline \multicolumn{9}{|l|}{$\begin{array}{l}\text { Sub-Saharan } \\
\text { Africa }\end{array}$} \\
\hline Guinea & $83.4[80.6,86.0]$ & $\begin{array}{l}84.1 \\
{[78.4,88.6]}\end{array}$ & $\begin{array}{l}84.3 \\
{[79.4,88.3]}\end{array}$ & $\begin{array}{l}78.3 \\
{[72.4,83.2]}\end{array}$ & $\begin{array}{l}85.8 \\
{[81.5,89.2]}\end{array}$ & $\begin{array}{l}84.7 \\
{[79.5,88.7]}\end{array}$ & $0.5[-5.4,6.5]$ & $\begin{array}{l}1.0 \\
{[0.9,1.1]}\end{array}$ \\
\hline Chad & $77.0[74.6,79.2]$ & $\begin{array}{l}77.9 \\
{[74.5,81.0]}\end{array}$ & $\begin{array}{l}79.8 \\
{[76.3,82.9]}\end{array}$ & $\begin{array}{l}77.9 \\
{[74.1,81.2]}\end{array}$ & $\begin{array}{l}77.6 \\
{[73 \cdot 6,81.1]}\end{array}$ & $\begin{array}{l}71.8 \\
{[67.2,76.0]}\end{array}$ & $-6.2[-11.6,-0.7]$ & $\begin{array}{l}0.9 \\
{[0.8,1.0]}\end{array}$ \\
\hline Cote d'Ivoire & $69.2[65.7,72.5]$ & $\begin{array}{l}52.9 \\
{[48.6,57.2]}\end{array}$ & $\begin{array}{l}49.5 \\
{[44.7,54.3]}\end{array}$ & $\begin{array}{l}41.9 \\
{[37.1,46.8]}\end{array}$ & $\begin{array}{l}48.3 \\
{[43.4,53.1]}\end{array}$ & $\begin{array}{l}47.9 \\
{[41.8,54.0]}\end{array}$ & $0.2[-8.3,8.6]$ & $\begin{array}{l}1.0 \\
{[0.9,1.1]}\end{array}$ \\
\hline Cameroon & $68.8[66.1,71.4]$ & $\begin{array}{l}78.3 \\
{[73.6,82.3]}\end{array}$ & $\begin{array}{l}68.5 \\
{[63.1,73.4]}\end{array}$ & $\begin{array}{l}70.7 \\
{[65.5,75.5]}\end{array}$ & $\begin{array}{l}60.7 \\
{[55.5,65.8]}\end{array}$ & $\begin{array}{l}61.5 \\
{[56.6,66.1]}\end{array}$ & $\begin{array}{l}-16.8 \\
{[-23.2,-10.4]}\end{array}$ & $\begin{array}{l}0.8 \\
{[0.7,0.9]}\end{array}$ \\
\hline Gabon & $67.7[63.5,71.5]$ & $\begin{array}{l}65.3 \\
{[59.2,70.9]}\end{array}$ & $\begin{array}{l}67.8 \\
{[60.6,74.2]}\end{array}$ & $\begin{array}{l}69.1 \\
{[61.2,76.0]}\end{array}$ & $\begin{array}{l}60.8 \\
{[51.9,69.0]}\end{array}$ & $\begin{array}{l}75.5 \\
{[67.3,82.2]}\end{array}$ & $10.3[0.9,19.6]$ & $\begin{array}{l}1.2 \\
{[1.0,1.3]}\end{array}$ \\
\hline Nigeria & $66.8[65.0,68.6]$ & $\begin{array}{l}69.9 \\
{[66.4,73.3]}\end{array}$ & $\begin{array}{l}72.4 \\
{[69.2,75.5]}\end{array}$ & $\begin{array}{l}66.5 \\
{[63.6,69.2]}\end{array}$ & $\begin{array}{l}63.1 \\
{[60.5,65.6]}\end{array}$ & $\begin{array}{l}62.1 \\
{[59.3,64.9]}\end{array}$ & $-7.8[-12.2,-3.4]$ & $\begin{array}{l}0.9 \\
{[0.8,0.9]}\end{array}$ \\
\hline Senegal & $66.4[64.2,68.6]$ & $\begin{array}{l}63.2 \\
{[59.0,67.1]}\end{array}$ & $\begin{array}{l}65.0 \\
{[61.3,68.5]}\end{array}$ & $\begin{array}{l}65.0 \\
{[60.9,68.8]}\end{array}$ & $\begin{array}{l}67.4 \\
{[62.6,71.8]}\end{array}$ & $\begin{array}{l}71.7 \\
{[67.1,75.9]}\end{array}$ & $8.6[2.6,14.6]$ & $\begin{array}{l}1.1 \\
{[1.0,1.2]}\end{array}$ \\
\hline Comoros & $66.3[62.1,70.2]$ & $\begin{array}{l}67.2 \\
{[58.5,74.8]}\end{array}$ & $\begin{array}{l}67.0 \\
{[60.2,73.3]}\end{array}$ & $\begin{array}{l}62.4 \\
{[55.1,69.2]}\end{array}$ & $\begin{array}{l}73.3 \\
{[65.8,79.6]}\end{array}$ & $\begin{array}{l}61.5 \\
{[52.9,69.4]}\end{array}$ & $-5.7[-17.1,5.6]$ & $\begin{array}{l}0.9 \\
{[0.7,1.1]}\end{array}$ \\
\hline Sao Tome & $61.7[56.5,66.7]$ & $\begin{array}{l}51.6 \\
{[43.5,59.8]}\end{array}$ & $\begin{array}{l}64.4 \\
{[55.7,72.3]}\end{array}$ & $\begin{array}{l}61.3 \\
{[51.9,69.9]}\end{array}$ & $\begin{array}{l}63.7 \\
{[54.0,72.5]}\end{array}$ & $\begin{array}{l}69.3 \\
{[58.9,78.1]}\end{array}$ & $17.7[5.4,29.9]$ & $\begin{array}{l}1.3 \\
{[1.1,1.6]}\end{array}$ \\
\hline Angola & $51.7[48.9,54.4]$ & $\begin{array}{l}50.5 \\
{[46.3,54.7]}\end{array}$ & $\begin{array}{l}53.4 \\
{[48.8,57.8]}\end{array}$ & $\begin{array}{l}52.6 \\
{[48.2,57.0]}\end{array}$ & $\begin{array}{l}51.7 \\
{[47.2,56.1]}\end{array}$ & $\begin{array}{l}50.3 \\
{[44.1,56.5]}\end{array}$ & $-0.2[-7.6,7.2]$ & $\begin{array}{l}1.0 \\
{[0.8,1.1]}\end{array}$ \\
\hline Tanzania & $48.7[46.0,51.4]$ & $\begin{array}{l}48.2 \\
{[43.3,53.1]}\end{array}$ & $\begin{array}{l}50.7 \\
{[45.9,55.6]}\end{array}$ & $\begin{array}{l}52.2 \\
{[46.8,57.5]}\end{array}$ & $\begin{array}{l}46.9 \\
{[42.5,51.4]}\end{array}$ & $\begin{array}{l}45.6 \\
{[41.6,49.7]}\end{array}$ & $-2.5[-8.8,3.7]$ & $\begin{array}{l}0.9 \\
{[0.8,1.1]}\end{array}$ \\
\hline Gambia & $48.5[43.9,53.1]$ & $\begin{array}{l}41.5 \\
{[35.2,48.1]}\end{array}$ & $\begin{array}{l}46.1 \\
{[39.9,52.5]}\end{array}$ & $\begin{array}{l}54.3 \\
{[48.3,60.2]}\end{array}$ & $\begin{array}{l}49.4 \\
{[42.2,56.5]}\end{array}$ & $\begin{array}{l}51.1 \\
{[43.5,58.6]}\end{array}$ & $9.6[1.1,18.1]$ & $\begin{array}{l}1.2 \\
{[1.1,1.5]}\end{array}$ \\
\hline $\begin{array}{l}\text { Democratic } \\
\text { Republic of } \\
\text { Congo }\end{array}$ & $48.1[45.3,50.9]$ & $\begin{array}{l}69.5 \\
{[62.5,75.8]}\end{array}$ & $\begin{array}{l}68.4 \\
{[61.6,74.4]}\end{array}$ & $\begin{array}{l}70.1 \\
{[64.7,75.0]}\end{array}$ & $\begin{array}{l}68.2 \\
{[61.7,74.1]}\end{array}$ & $\begin{array}{l}69.7 \\
{[64.1,74.8]}\end{array}$ & $-5.1[-12.5,2.3]$ & $\begin{array}{l}0.9 \\
{[0.8,1.1]}\end{array}$ \\
\hline Niger & $47.1[44.2,49.9]$ & $\begin{array}{l}48.1 \\
{[43.5,52.8]}\end{array}$ & $\begin{array}{l}47.5 \\
{[42.7,52.4]}\end{array}$ & $\begin{array}{l}51.1 \\
{[46.2,55.9]}\end{array}$ & $\begin{array}{l}49.6 \\
{[44.0,55.1]}\end{array}$ & $\begin{array}{l}39.0 \\
{[34.7,43.5]}\end{array}$ & $-9.1[-15.3,-2.9]$ & $\begin{array}{l}0.8 \\
{[0.7,0.9]}\end{array}$ \\
\hline Sierra Leone & $46.2[42.6,49.8]$ & $\begin{array}{l}45.7 \\
{[39.5,52.1]}\end{array}$ & $\begin{array}{l}42.4 \\
{[37.4,47.6]}\end{array}$ & $\begin{array}{l}45.1 \\
{[40.2,50.2]}\end{array}$ & $\begin{array}{l}47.6 \\
{[41.9,53.4]}\end{array}$ & $\begin{array}{l}50.0 \\
{[44.6,55.4]}\end{array}$ & $4.3[-3.7,12.4]$ & $\begin{array}{l}1.1 \\
{[0.9,1.3]}\end{array}$ \\
\hline Benin & $45.9[43.7,48.2]$ & $\begin{array}{l}46.7 \\
{[42.7,50.7]}\end{array}$ & $\begin{array}{l}44.3 \\
{[40.6,48.1]}\end{array}$ & $\begin{array}{l}42.1 \\
{[38.3,46.1]}\end{array}$ & $\begin{array}{l}49.1 \\
{[44.9,53.3]}\end{array}$ & $\begin{array}{l}47.4 \\
{[43.5,51.3]}\end{array}$ & $0.7[-4.8,6.2]$ & $\begin{array}{l}1.0 \\
{[0.9,1.1]}\end{array}$ \\
\hline Ghana & $44.4[41.4,47.5]$ & $\begin{array}{l}42.1 \\
{[36.8,47.6]}\end{array}$ & $\begin{array}{l}42.8 \\
{[37.0,48.8]}\end{array}$ & $\begin{array}{l}44.6 \\
{[38.3,51.0]}\end{array}$ & $\begin{array}{l}45.0 \\
{[39.5,50.7]}\end{array}$ & $\begin{array}{l}47.4 \\
{[40.8,54.2]}\end{array}$ & $5.3[-3.4,14]$ & $\begin{array}{l}1.1 \\
{[0.9,1.3]}\end{array}$ \\
\hline Zimbabwe & $42.4[39.4,45.4]$ & $\begin{array}{l}40.4 \\
{[34.5,46.5]}\end{array}$ & $\begin{array}{l}40.4 \\
{[34.5,46.5]}\end{array}$ & $\begin{array}{l}44.0 \\
{[39.1,49.1]}\end{array}$ & $\begin{array}{l}43.9 \\
{[38.5,49.5]}\end{array}$ & $\begin{array}{l}43.2 \\
{[37.6,49.0]}\end{array}$ & $2.9[-5.4,11.1]$ & $\begin{array}{l}1.1 \\
{[0.9,1.3]}\end{array}$ \\
\hline Mali & $42.2[39.7,44.8]$ & $\begin{array}{l}43.1 \\
{[38.7,47.5]}\end{array}$ & $\begin{array}{l}42.5 \\
{[38.3,46.7]}\end{array}$ & $\begin{array}{l}42.3 \\
{[37.7,47.0]}\end{array}$ & $\begin{array}{l}39.5 \\
{[35.1,44.1]}\end{array}$ & $\begin{array}{l}43.9 \\
{[38.2,49.7]}\end{array}$ & $0.8[-6.3,7.9]$ & $\begin{array}{l}1.0 \\
{[0.8,1.2]}\end{array}$ \\
\hline Togo & $39.4[36.5,42.4]$ & $\begin{array}{l}39.9 \\
{[35.2,44.9]}\end{array}$ & $\begin{array}{l}46.1 \\
{[41.1,51.3]}\end{array}$ & $\begin{array}{l}43.6 \\
{[38.7,48.6]}\end{array}$ & $\begin{array}{l}34.9 \\
{[30.2,39.9]}\end{array}$ & $\begin{array}{l}32.3 \\
{[27.4,37.7]}\end{array}$ & $-7.6[-14.4,-0.8]$ & $\begin{array}{l}0.8 \\
{[0.6,1.0]}\end{array}$ \\
\hline Liberia & $38.8[34.9,42.7]$ & $\begin{array}{l}38.7 \\
{[32.9,44.8]}\end{array}$ & $\begin{array}{l}40.4 \\
{[34.8,46.3]}\end{array}$ & $\begin{array}{l}32.6 \\
{[27.3,38.5]}\end{array}$ & $\begin{array}{l}35.7 \\
{[27.9,44.4]}\end{array}$ & $\begin{array}{l}46.4 \\
{[39.4,53.6]}\end{array}$ & $7.7[-1.5,16.9]$ & $\begin{array}{l}1.2 \\
{[0.9,1.5]}\end{array}$ \\
\hline Lesotho & $34.7[31.6,37.9]$ & $\begin{array}{l}28.6 \\
{[23.0,35.0]}\end{array}$ & $\begin{array}{l}38.3 \\
{[32.2,44.8]}\end{array}$ & $\begin{array}{l}30.9 \\
{[24.6,38.1]}\end{array}$ & $\begin{array}{l}34.8 \\
{[29.2,41.0]}\end{array}$ & $\begin{array}{l}40.9 \\
{[33.5,48.6]}\end{array}$ & $12.2[2.5,22.0]$ & $\begin{array}{l}1.4 \\
{[1.0,1.8]}\end{array}$ \\
\hline
\end{tabular}




\begin{tabular}{|lllllllll|} 
Zambia & $34.2[32.1,36.4]$ & 35.5 & 38.4 & 33.3 & 30.2 & 33.6 & $-1.9[-7.1,3.2]$ & 0.9 \\
& & {$[32.1,39.1]$} & {$[34.5,42.4]$} & {$[29.6,37.2]$} & {$[26.2,34.5]$} & {$[29.8,37.6]$} & {$[0.8,1.1]$} \\
\hline Uganda & $33.9[32.1,35.8]$ & 41.3 & 36.0 & 32.7 & 31.6 & 28.1 & -13.2 & 0.7 \\
& & {$[37.7,45.1]$} & {$[32.8,39.3]$} & {$[29.5,36.0]$} & {$[28.2,35.1]$} & {$[24.3,32.3]$} & {$[-18.7,-7.8]$} & {$[0.6,0.8]$} \\
\hline South Africa & $32.7[29.5,36.1]$ & 33.3 & 27.0 & 32.0 & 34.8 & 36.5 & $3.3[-7.1,13.6]$ & 1.1 \\
& & {$[26.7,40.6]$} & {$[21.1,33.7]$} & {$[26.1,38.6]$} & {$[28.7,41.4]$} & {$[29.3,44.4]$} & {$[0.8,1.4]$} \\
\hline Sudan & $31.3[29.3,33.4]$ & 37.9 & 30.2 & 26.9 & 31.8 & 28.7 & $-9.2[-15.9,-2.5]$ & 0.8 \\
& & {$[33.2,42.8]$} & {$[27.0,33.6]$} & {$[23.5,30.5]$} & {$[28.1,35.7]$} & {$[24.4,33.5]$} & {$[0.6,0.9]$} \\
\hline Namibia & $28.8[26.6,31.1]$ & 27.7 & 28.0 & 27.2 & 27.2 & 33.8 & $6.1[-1.1,13.3]$ & 1.2 \\
& & {$[22.8,33.3]$} & {$[23.4,33.1]$} & {$[22.5,32.5]$} & {$[22.7,32.2]$} & {$[28.9,39.0]$} & & {$[0.9,1.5]$} \\
\hline Ethiopia & $26.7[24.4,29.1]$ & 27.7 & 24.7 & 25.1 & 32.1 & 23.7 & $-4.0[-10.6,2.6]$ & 0.9 \\
& & {$[22.9,33.1]$} & {$[19.6,30.6]$} & {$[20.6,30.1]$} & {$[27.8,36.7]$} & {$[19.7,28.2]$} & & {$[0.6,1.1]$} \\
\hline Malawi & $23.8[22.1,25.5]$ & 24.6 & 21.0 & 20.3 & 25.2 & 27.6 & $3.0[-1.3,7.2]$ & 1.1 \\
& & {$[21.6,27.9]$} & {$[18.2,24.2]$} & {$[17.4,23.6]$} & {$[22.0,28.6]$} & {$[24.3,31.2]$} & & {$[0.9,1.3]$} \\
\hline Rwanda & $19.5[18.0,21.2]$ & 18.6 & 20.9 & 17.4 & 19.4 & 21.4 & $2.8[-1.7,7.3]$ & 1.1 \\
& & {$[15.7,21.8]$} & {$[17.6,24.7]$} & {$[14.5,20.7]$} & {$[16.3,22.9]$} & {$[18.2,24.9]$} & & {$[0.9,1.4]$} \\
\hline Burundi & $15.0[13.8,16.2]$ & 16.0 & 12.2 & 13.9 & 15.8 & 16.7 & $0.7[-3.1,4.5]$ & 1.0 \\
& & {$[13.4,19.1]$} & {$[10.0,14.9]$} & {$[11.7,16.6]$} & {$[13.6,18.4]$} & {$[14.5,19.2]$} & & {$[0.8,1.3]$} \\
\hline
\end{tabular}

A positive value of absolute difference was obtained for 37 study countries, suggesting a pro-poor inequality where the prevalence of delayed breastfeeding initiation was higher among the wealthiest than among the poorest. Most of these countries are in Sub-Saharan Africa. The positive absolute difference was higher than 20 percentage points in Thailand, Guyana, and Guatemala. On the other hand, for 21 study countries, we obtained a negative value which denotes a pro-rich inequality where the prevalence of delayed breastfeeding initiation was higher among the poorest than in the wealthiest. The negative value for the absolute difference was less than ten percentage points in four countries, namely, Ukraine, Maldives, Cameroon, Uganda.

Figure 1 presents the median, interquartile range and the variation in the prevalence of breastfeeding initiation across all wealth quintiles for all countries and by region. In all countries combined, the median prevalence of delayed initiation was $42.6 \%$ [interquartile range $(\mathrm{IQR})=17.4 \%$ ] among the poorest quintile, compared to $47.4 \%$ [IQR $=27.7 \%$ ] among the wealthiest. The highest median prevalence of delayed initiation in the wealthiest quintile was $61.5 \%$ [IQR $=12.1 \%]$ in Latin America and the Caribbean, 58.1\% [IQR = 27.9\%] in the Middle East and North Africa, and 55.5\% [IQR = 11.0\%] in South Asia. The median prevalence of delayed breastfeeding initiation in the poorest quintile was lower than the wealthiest, with the highest prevalence of $51.2 \%$ [IQR $=$ 21.3\%] in South Asia, followed by 49.9\% [IQR $=24.7 \%]$ in the Middle East and North Africa, and 45.3\% [IQR $=15.9 \%$ in Latin America and Caribbean.

In Fig. 2, we plotted the absolute inequality in delayed breastfeeding initiation against the national prevalence. There was no distinguishable clustering of countries by world region. The 'zero-line indicates no inequality. Twelve countries were within $₫ 2.5$ percent points of the zero-line indicating low or no absolute inequality in delayed breastfeeding initiation. Of the 12 countries, eight are in Sub-Saharan Africa, two in South Asia, and one in Europe and Central Asia and East Asia and the Pacific. Four countries (Gabon, Sao Tome, Thailand and Tunisia) demonstrated a high positive value for absolute inequality ( $\geq 10$ percent points) alongside a high national prevalence $(\geq 60 \%)$, indicating a high pro-poor inequality. Conversely, Cameroon reported a high negative value for absolute inequality ( $\geq-10$ percent points) alongside a high national prevalence $(\geq 60 \%)$ indicating a high pro-rich inequality. All study countries in Latin America and the Caribbean had a high positive value for absolute inequality, with delayed initiation more prevalent among the wealthiest quintile.

Figure 3 shows the pattern between absolute inequality in the prevalence of delayed initiation of breastfeeding and the overall income inequality in the countries. The correlation coefficient $(r)$ between the country-level income inequality and absolute inequality in the prevalence of delayed breastfeeding initiation was very low $(r=0.09)$.

Table 2 presents the stratified prevalence of delayed breastfeeding initiation across the poorest and wealthiest quintiles by place of residence. In 25 study countries, the prevalence of delayed initiation was higher among the wealthiest quintile in urban and rural areas. In seven countries, the prevalence was higher among the poorest in the rural areas, with an absolute inequality of more than - 10 percent points. In contrast, in three of these countries (Cameroon, Uganda, Yemen), the wealthiest quintile in the urban areas had higher delayed initiation. Cameroon showed the steepest gradient across the wealth quintiles in rural areas with a percentage point difference of -40.4 between the wealthiest and poorest. At the same time, Sudan had the steepest gradient of -29.2 percent points in urban areas. On the other hand, Cambodia (percent point difference 33.5) and Guyana (percent point difference 28.2) had the steepest gradient in urban and rural areas, respectively, where delayed initiation was more prevalent among the wealthiest quintile.

Table 2: Delayed breastfeeding initiation rates by economic status and place of residence in 58 low-and middle-income countries, by World Bank region (2012-17). Values are percentages (95\% confidence intervals) 
Page $8 / 20$ 


\begin{tabular}{|c|c|c|c|c|c|c|}
\hline \multirow[t]{3}{*}{ Region/Country } & \multicolumn{3}{|l|}{ Urban } & \multicolumn{3}{|l|}{ Rural } \\
\hline & \multirow[t]{2}{*}{$\begin{array}{l}\text { Prevalence of delayed } \\
\text { initiation of breastfeeding }\end{array}$} & \multicolumn{2}{|c|}{$\begin{array}{l}\text { Prevalence of delayed initiation } \\
\text { across wealth quintiles }\end{array}$} & \multirow[t]{2}{*}{$\begin{array}{l}\text { Prevalence of delayed } \\
\text { initiation of breastfeeding }\end{array}$} & \multicolumn{2}{|c|}{$\begin{array}{l}\text { Prevalence of delayed initiation } \\
\text { across wealth quintiles }\end{array}$} \\
\hline & & $\begin{array}{l}\text { 1st fifth } \\
\text { (poorest) }\end{array}$ & $\begin{array}{l}\text { 5th fifth } \\
\text { (wealthiest) }\end{array}$ & & $\begin{array}{l}\text { 1st fifth } \\
\text { (poorest) }\end{array}$ & $\begin{array}{l}\text { 5th fifth } \\
\text { (wealthiest) }\end{array}$ \\
\hline \multicolumn{7}{|l|}{$\begin{array}{l}\text { East Asia \& } \\
\text { Pacific }\end{array}$} \\
\hline Thailand & $63.0[56.6,68.9]$ & $50.8[32.6,68.8]$ & $75.5[64.2,84.2]$ & $57.7[51.4,63.8]$ & $45.9[36.6,55.5]$ & $68.3[47.0,83.9]$ \\
\hline Indonesia & $43.2[40.9,45.5]$ & $47.4[43.4,51.4]$ & $37.7[31.3,44.6]$ & $43.7[41.1,46.2]$ & $43.7[39.6,47.9]$ & $46.5[41.5,51.5]$ \\
\hline Philippines & $44.4[40.1,48.9]$ & $40.7[34.3,47.4]$ & $57.6[42.4,71.4]$ & $42.0[38.5,45.6]$ & $36.6[30.7,42.9]$ & $47.6[40.0,55.3]$ \\
\hline Cambodia & $49.4[43.8,55.0]$ & $28.7[21.5,37.1]$ & $62.2[53.2,70.6]$ & $35.5[32.6,38.5]$ & $37.6[32.1,43.5]$ & $41.9[34.8,49.4]$ \\
\hline Myanmar & $30.2[24.3,36.9]$ & $36.6[24.6,50.5]$ & $33.4[22.4,46.4]$ & $34.2[30.6,38.0]$ & $34.9[27.4,43.3]$ & $29.7[23.4,37.0]$ \\
\hline Timor Leste & $26.4[21.3,32.1]$ & $38.6[30.2,47.8]$ & $22.8[15.6,32.2]$ & $24.1[21.2,27.4]$ & $27.9[21.7,35.1]$ & $24.5[19.5,30.2]$ \\
\hline \multicolumn{7}{|l|}{$\begin{array}{l}\text { Europe \& Central } \\
\text { Asia }\end{array}$} \\
\hline Armenia & $64.7[58.3,70.5]$ & $69.3[51.8,82.6]$ & $63.6[49.6,75.6]$ & $51.3[43.5,59.0]$ & $49.1[34.2,64.2]$ & $55.7[43.0,67.8]$ \\
\hline Albania & $38.8[31.6,46.5]$ & $25.0[14.3,39.9]$ & $49.1[30.3,68.2]$ & $49.7[44.1,55.3]$ & $61.1[50.0,71.1]$ & $43.5[33.5,54.0]$ \\
\hline Moldova & $41.2[35.9,46.7]$ & $44.1[17.4,74.7]$ & $38.4[32.4,44.8]$ & $37.7[32.1,43.7]$ & $43.5[31.5,56.3]$ & $39.6[19.1,64.4]$ \\
\hline Tajikistan & $37.0[31.6,42.7]$ & $33.1[25.6,41.6]$ & $35.6[25.9,46.7]$ & $38.7[34.7,42.9]$ & $32.7[27.2,38.6]$ & $43.9[33.1,55.3]$ \\
\hline Ukraine & $33.7[28.9,38.9]$ & $48.0[31.9,64.5]$ & $26.5[19.9,34.5]$ & $35.7[29.8,42.1]$ & $35.5[27.0,45.0]$ & $50.8[46.3,55.2]$ \\
\hline Kazakhstan & $17.0[14.6,19.8]$ & $12.6[7.4,20.6]$ & 13.5[9.6,18.7] & $16.4[13.4,19.9]$ & $14.5[10.1,20.4]$ & $31.5[10.3,64.8]$ \\
\hline Kyrgyz Republic & 18.7[14.3,24.0] & $17.3[10.0,28.4]$ & $19.1[11.4,30.4]$ & $15.2[12.8,17.9]$ & $9.1[5.5,14.6]$ & $23.8[17.6,31.3]$ \\
\hline \multicolumn{7}{|l|}{$\begin{array}{l}\text { Latin America } \\
\text { and the } \\
\text { Caribbean }\end{array}$} \\
\hline $\begin{array}{l}\text { Dominican } \\
\text { Republic }\end{array}$ & $57.3[52.6,61.8]$ & $49.9[43.3,56.5]$ & $64.1[51.4,75.2]$ & $55.7[45.1,65.7]$ & $43.6[32.5,55.3]$ & $61.8[44.7,76.4]$ \\
\hline Haiti & $56.5[52.1,60.7]$ & $55.1[47.2,62.8]$ & $63.4[54.5,71.5]$ & $50.7[47.6,53.9]$ & $48.4[42.6,54.2]$ & $57.9[51.1,64.4]$ \\
\hline Guyana & $57.2[49.0,64.9]$ & $56.8[40.7,71.5]$ & $79.2[67.0,87.7]$ & $48.8[44.5,53.1]$ & $39.9[33.6,46.6]$ & $68.1[56.9,77.5]$ \\
\hline Guatemala & $46.6[43.4,49.9]$ & $30.7[25.6,36.3]$ & $57.0[50.5,63.2]$ & $31.6[29.2,34.2]$ & $21.8[18.1,26.1]$ & $47.8[42.2,53.4]$ \\
\hline \multicolumn{7}{|l|}{$\begin{array}{l}\text { The Middle East } \\
\text { \& North Africa }\end{array}$} \\
\hline Egypt & $75.6[72.8,78.2]$ & $74.6[68.0,80.3]$ & $77.9[71.0,83.6]$ & 71.7[69.5,73.8] & $69.2[65.7,72.5]$ & $73.8[69.5,77.6]$ \\
\hline Tunisia & $63.9[59.2,68.4]$ & $65.4[49.5,78.4]$ & $69.1[59.1,77.6]$ & $53.5[46.8,60.1]$ & $51.8[44.1,59.3]$ & $55.8[21.0,85.7]$ \\
\hline Yemen & $44.8[40.1,49.5]$ & $48.1[37.8,58.6]$ & $41.7[31.9,52.2]$ & $48.2[45.7,50.7]$ & $44.9[40.1,49.9]$ & $30.5[25.5,36.0]$ \\
\hline Jordan & $32.6[29.9,35.5]$ & $30.2[23.8,37.4]$ & $38.8[33.3,44.7]$ & $35.4[29.7,41.5]$ & - & $37.6[32.3,43.3]$ \\
\hline \multicolumn{7}{|l|}{ South Asia } \\
\hline Pakistan & $79.2[75.0,82.9]$ & $87.9[80.4,92.8]$ & $66.2[51.9,78.1]$ & $81.0[77.7,84.0]$ & $74.7[64.8,82.7]$ & $85.3[81.2,88.7]$ \\
\hline Afghanistan & $62.3[57.9,66.6]$ & $60.5[52.4,68.1]$ & $66.1[58.9,72.6]$ & $58.1[54.7,61.3]$ & $56.1[52.1,60.1]$ & $56.3[52.0,60.5]$ \\
\hline India & $57.1[55.8,58.3]$ & $53.8[51.4,56.2]$ & $57.8[54.8,60.7]$ & $59.0[58.5,59.6]$ & $60.8[59.7,61.9]$ & $56.9[55.7,58.0]$ \\
\hline Bangladesh & $54.7[49.9,59.5]$ & $47.1[39.6,54.6]$ & $64.1[56.5,71.1]$ & $47.2[44.1,50.4]$ & $44.5[37.3,52.0]$ & $48.2[42.6,53.8]$ \\
\hline Nepal & $43.0[39.0,47.1]$ & $38.7[32.2,45.6]$ & $46.0[38.4,53.7]$ & $47.5[42.9,52.1]$ & $33.9[26.1,42.7]$ & $53.2[44.9,61.3]$ \\
\hline Maldives & $43.1[33.9,52.7]$ & $63.5[44.6,79.0]$ & $44.4[25.8,64.7]$ & $27.8[24.7,31.1]$ & $25.2[20.0,31.1]$ & $21.7[17.0,27.3]$ \\
\hline \multicolumn{7}{|l|}{$\begin{array}{l}\text { Sub-Saharan } \\
\text { Africa }\end{array}$} \\
\hline Guinea & $81.4[77.4,84.8]$ & $84.4[74.4,91.0]$ & $83.1[73.9,89.6]$ & $84.2[80.5,87.3]$ & $84.1[77.1,89.2]$ & $85.3[78.7,90.1]$ \\
\hline Chad & $78.1[74.2,81.6]$ & $81.6[75.0,86.8]$ & $75.0[68.4,80.5]$ & $76.7[73.9,79.4]$ & $76.8[72.8,80.4]$ & $70.4[64.4,75.8]$ \\
\hline
\end{tabular}

Page 9/20 


\begin{tabular}{|c|c|c|c|c|c|c|}
\hline Cote d'Ivoire & $52.4[48.2,56.6]$ & $57.8[51.7,63.6]$ & $48.6[42.3,54.8]$ & $46.1[42.5,49.8]$ & $48.9[43.1,54.8]$ & $47.1[36.1,58.3]$ \\
\hline Cameroon & $66.6[63.0,70.1]$ & $83.3[66.4,92.7]$ & 62.3[57.4,67.0] & $70.3[66.5,73.8]$ & $77.9[73.1,82.1]$ & $37.5[18.5,61.2]$ \\
\hline Gabon & $67.6[62.8,72.1]$ & $67.0[59.2,74.0]$ & $76.2[67.0,83.5]$ & $68.0[63.5,72.2]$ & $60.1[52.4,67.3]$ & $70.4[60.8,78.4]$ \\
\hline Nigeria & $59.9[57.2,62.6]$ & $59.7[53.8,65.4]$ & $65.7[61.2,70.0]$ & $70.6[68.4,72.7]$ & $74.6[70.7,78.2]$ & $59.4[55.9,62.9]$ \\
\hline Senegal & $68.0[64.4,71.3]$ & $62.6[56.0,68.7]$ & 75.3[67.4,81.8] & $65.6[62.7,68.4]$ & $63.3[58.3,68.1]$ & $69.4[63.6,74.7]$ \\
\hline Comoros & $71.2[64.3,77.2]$ & $65.7[49.2,79.2]$ & $74.5[61.6,84.2]$ & $64.4[59.2,69.2]$ & $67.7[57.4,76.6]$ & $57.0[47.6,65.9]$ \\
\hline Sao Tome & $64.7[58.1,70.7]$ & $48.9[38.8,59.0]$ & $70.6[59.8,79.6]$ & $56.2[47.5,64.5]$ & $55.2[41.9,67.8]$ & $56.4[27.0,81.8]$ \\
\hline Angola & $49.9[46.2,53.6]$ & $52.7[47.5,57.8]$ & $49.8[42.6,57.0]$ & $54.4[50.3,58.4]$ & $46.5[39.6,53.6]$ & $52.3[41.4,63.0]$ \\
\hline Tanzania & $45.7[40.8,50.7]$ & $47.3[37.1,57.7]$ & $47.0[41.1,52.9]$ & $49.9[46.7,53.1]$ & $48.5[43.1,54.0]$ & $44.3[38.9,49.9]$ \\
\hline Gambia & $52.8[45.5,59.9]$ & $39.4[29.6,50.2]$ & $60.1[50.0,69.5]$ & $44.8[39.4,50.3]$ & 43.5[35.9,51.4] & $43.7[34.2,53.7]$ \\
\hline $\begin{array}{l}\text { Democratic } \\
\text { Republic of } \\
\text { Congo }\end{array}$ & $66.9[61.6,71.8]$ & $55.0[42.4,67.1]$ & $68.7[58.5,77.4]$ & $70.6[65.8,75.0]$ & $75.4[68.1,81.5]$ & $70.5[64.1,76.1]$ \\
\hline Niger & $28.2[23.8,33.1]$ & $29.6[23.0,37.2]$ & $29.9[24.1,36.5]$ & $50.0[46.9,53.1]$ & $54.3[48.8,59.6]$ & $41.4[36.2,46.8]$ \\
\hline Sierra Leone & $57.1[51.4,62.6]$ & $54.9[44.5,65.0]$ & 63.7[55.8,71.0] & $42.4[38.2,46.8]$ & $42.7[35.4,50.3]$ & $42.3[36.1,48.8]$ \\
\hline Benin & $47.2[43.7,50.6]$ & $48.7[42.9,54.6]$ & $47.1[41.6,52.7]$ & $45.2[42.3,48.1]$ & $44.8[39.6,50.2]$ & $47.6[42.2,53.2]$ \\
\hline Ghana & $45.5[41.2,49.8]$ & $42.6[34.7,50.8]$ & $51.0[39.4,62.5]$ & $43.5[39.3,47.8]$ & $41.8[34.8,49.1]$ & $45.2[37.9,52.7]$ \\
\hline Zimbabwe & $45.9[41.8,50.0]$ & $43.6[34.2,53.5]$ & $49.4[41.8,57.0]$ & $41.0[37.1,45.0]$ & $38.7[31.3,46.7]$ & $49.4[41.8,57.0]$ \\
\hline Mali & $38.3[34.1,42.6]$ & $40.5[32.6,48.8]$ & $37.2[29.9,45.2]$ & $43.3[40.3,46.3]$ & $44.0[38.8,49.3]$ & $45.8[38.8,53.0]$ \\
\hline Togo & $38.4[33.6,43.4]$ & $38.7[30.9,47.1]$ & $43.9[34.9,53.4]$ & $39.9[36.3,43.6]$ & $40.8[35.1,46.8]$ & $26.6[21.5,32.4]$ \\
\hline Liberia & $37.1[31 \cdot 0,43.5]$ & $31.4[23 \cdot 0,41.3]$ & $45.2[36.2,54.5]$ & $40.5[36.0,45.1]$ & $44.4[37.2,51.7]$ & $48.4[37.7,59.3]$ \\
\hline Lesotho & $36.3[29.5,43.7]$ & $46.3[31.6,61.7]$ & $41.0[26.9,56.8]$ & $34.1[30.8,37.7]$ & $24.6[19.2,31.0]$ & $40.8[32.6,49.6]$ \\
\hline Zambia & $32.7[29.2,36.3]$ & $32.3[27.4,37.6]$ & $35.4[30.3,40.9]$ & $34.9[32.3,37.7]$ & $37.0[32.6,41.7]$ & $39.4[32.0,47.3]$ \\
\hline Uganda & $28.9[25.0,33.1]$ & $31.2[24.2,39.3]$ & $27.7[20.6,36.0]$ & $35.3[33.3,37.4]$ & $44.8[41.0,48.7]$ & $28.3[23.9,33.2]$ \\
\hline South Africa & $30.4[26.2,35.0]$ & $34.2[24.7,45.2]$ & $33.4[24.4,43.7]$ & $36.6[32.1,41.2]$ & $32.0[24.0,41.2]$ & $43.5[32.8,54.8]$ \\
\hline Sudan & $29.0[25.8,32.5]$ & $55.1[40.2,69.2]$ & $25.9[20.3,32.3]$ & $32.1[29.7,34.6]$ & $37.2[32.4,42.2]$ & $33.1[27.0,39.7]$ \\
\hline Namibia & $30.4[27.3,33.6]$ & $28.1[21.7,35.6]$ & $36.9[30.6,43.7]$ & $27.4[24.2,30.7]$ & $27.1[19.9,35.6]$ & $28.3[21.4,36.4]$ \\
\hline Ethiopia & $27.4[21.9,33.6]$ & 25.7[18.0,35.3] & $26.2[19.8,33.9]$ & $26.6[24.1,29.2]$ & $28.2[22.6,34.7]$ & $23.2[18.6,28.5]$ \\
\hline Malawi & $36.5[31.7,41.7]$ & $32.6[24.8,41.5]$ & $38.5[31.5,45.9]$ & $21.7[20.1,23.4]$ & 23.4[20.2,26.9] & $23.3[20.0,27.0]$ \\
\hline Rwanda & $21.7[18.2,25.8]$ & $16.4[11.2,23.5]$ & 23.0[18.6,28.1] & $19.1[17.4,20.9]$ & 19.1[15.8,22.9] & $20.5[16.5,25.3]$ \\
\hline Burundi & $22.1[18.5,26.1]$ & $23.3[15.3,33.9]$ & $20.6[15.7,26.5]$ & $14.2[13.0,15.6]$ & $14.9[12.3,18.0]$ & $15.8[13.3,18.7]$ \\
\hline
\end{tabular}

\section{Trend in inequality}

Table 3 presents the change in prevalence of delayed breastfeeding initiation at the national level and in the wealthiest and the poorest quintiles over time. For 41 out of 58 study countries, we retrieved data on the prevalence of delayed breastfeeding initiation from a ten-year previous survey round (between 2002 to 2007). Thirty-two countries had a positive value for annual absolute change indicating an overall increase in the prevalence, while nine countries had a negative value suggesting a decrease. The greatest annual absolute increase in delayed breastfeeding initiation over time was in the Dominican Republic and Egypt, with a 2.9 and 1.4 percentage point increase every year, respectively. The number of countries with a prevalence of delayed breastfeeding initiation more than $50 \%$ reduced from 28 in 2002-2007 to 13 in 2012-2017. At the same time, the largest annual decrease in prevalence was -4.8 percent points in Ukraine, -3.5 percent points in Togo and - 3.3 percent points in Yemen. Overall, the reduction was faster among the wealthiest than the poorest in 17 countries, with the excess reduction ranging from - 0.1 in Kazakhstan to -3.0 in the Democratic Republic of Congo. Overall, delayed breastfeeding initiation rates decreased faster in the poorest quintile (median - 1.3 percent points per year) compared to the wealthiest quintile (median 0.8 percent points per year). The largest annual absolute increase within the poorest quintile was in the Dominican Republic (2.8 percent points), and the largest increase within the wealthiest quintile was in Zimbabwe (1.8 percent points). The largest decrease was - 5.1 percent points in Ukraine in both the poorest and the wealthiest quintiles.

Table 3: Change over time in delayed initiation of breastfeeding rates by economic status in 41 low- and middle-income countries, by World Bank region (2002-07 [Year1], and 2012-17 [Year 2]). Values are percentages (95\% confidence intervals) 


\begin{tabular}{|c|c|c|c|c|c|c|c|c|c|c|}
\hline \multirow[t]{2}{*}{ Region/Country } & \multicolumn{3}{|c|}{ National Coverage } & \multicolumn{3}{|c|}{ 1st fifth (poorest) } & \multicolumn{3}{|c|}{ 5th fifth (wealthiest) } & \multirow{2}{*}{$\begin{array}{l}\text { Annual } \\
\text { absolute } \\
\text { excess } \\
\text { change } \\
\text { (percentage } \\
\text { points) }\end{array}$} \\
\hline & $\begin{array}{l}\text { Coverage } \\
\text { in Year } 2\end{array}$ & $\begin{array}{l}\text { Coverage } \\
\text { in Year } 1\end{array}$ & $\begin{array}{l}\text { Annual } \\
\text { Absolute } \\
\text { Change } \\
\text { (percent } \\
\text { points) }\end{array}$ & $\begin{array}{l}\text { Coverage } \\
\text { in Year } 2\end{array}$ & $\begin{array}{l}\text { Coverage } \\
\text { in Year } 1\end{array}$ & $\begin{array}{l}\text { Annual } \\
\text { Absolute } \\
\text { Change } \\
\text { (percent } \\
\text { points) }\end{array}$ & $\begin{array}{l}\text { Coverage } \\
\text { in Year } 2\end{array}$ & $\begin{array}{l}\text { Coverage } \\
\text { in Year } 1\end{array}$ & $\begin{array}{l}\text { Annual } \\
\text { Absolute } \\
\text { Change } \\
\text { (percent } \\
\text { points) }\end{array}$ & \\
\hline \multicolumn{11}{|l|}{$\begin{array}{l}\text { East Asia \& } \\
\text { Pacific }\end{array}$} \\
\hline Indonesia & $\begin{array}{l}43.4 \\
{[41.7,45.1]}\end{array}$ & $\begin{array}{l}59.8 \\
{[61.6,57.9]}\end{array}$ & $\begin{array}{l}-1.6 \\
{[-2.0,-1.3]}\end{array}$ & $\begin{array}{l}45.3 \\
{[42.4,48.2]}\end{array}$ & $\begin{array}{l}54.6 \\
{[57.9,51.3]}\end{array}$ & $\begin{array}{l}-0.9 \\
{[-1.6,-0.3]}\end{array}$ & $\begin{array}{l}43.5 \\
{[39.4,47.6]}\end{array}$ & $\begin{array}{l}63.3 \\
{[67.8,58.6]}\end{array}$ & $\begin{array}{l}-2.0 \\
{[-2.8,-1.1]}\end{array}$ & -1.1 \\
\hline Philippines & $\begin{array}{l}43.1 \\
{[40.4,45.9]}\end{array}$ & $\begin{array}{l}53.9 \\
{[56.3,51.6]}\end{array}$ & $\begin{array}{l}-0.8 \\
{[-1.1,-0.4]}\end{array}$ & $\begin{array}{l}38.3 \\
{[33.9,43.0]}\end{array}$ & $\begin{array}{l}48.5 \\
{[52.9,44.2]}\end{array}$ & $\begin{array}{l}-0.7 \\
{[-1.4,-0.1]}\end{array}$ & $\begin{array}{l}50.6 \\
{[43.4,57.8]}\end{array}$ & $\begin{array}{l}59.8 \\
{[65.6,53.8]}\end{array}$ & $\begin{array}{l}-0.7 \\
{[-1.6,0.3]}\end{array}$ & 0.1 \\
\hline Cambodia & $\begin{array}{l}37.4 \\
{[34.8,40.2]}\end{array}$ & $\begin{array}{l}64.5 \\
{[66.7,62.1]}\end{array}$ & $\begin{array}{l}-3.0 \\
{[-3.5,-2.4]}\end{array}$ & $\begin{array}{l}36.6 \\
{[31.6,41.8]}\end{array}$ & $\begin{array}{l}71.4 \\
{[75.4,67.1]}\end{array}$ & $\begin{array}{l}-3.9 \\
{[-4.9,-2.8]}\end{array}$ & $\begin{array}{l}47.1 \\
{[41.0,53.2]}\end{array}$ & $\begin{array}{l}59.5 \\
{[63.8,55.0]}\end{array}$ & $\begin{array}{l}-1.4 \\
{[-2.5,-0.2]}\end{array}$ & 2.5 \\
\hline \multicolumn{11}{|l|}{$\begin{array}{l}\text { Europe \& } \\
\text { Central Asia }\end{array}$} \\
\hline Armenia & $\begin{array}{l}59.1 \\
{[54.1,63.9]}\end{array}$ & $\begin{array}{l}67.8 \\
{[72.4,62.8]}\end{array}$ & $\begin{array}{l}-0.9 \\
{[-1.8,0.1]}\end{array}$ & $\begin{array}{l}61.7 \\
{[49.1,72.9]}\end{array}$ & $\begin{array}{l}78.5 \\
{[85.6,69.0]}\end{array}$ & $\begin{array}{l}-1.7 \\
{[-3.7,0.4]}\end{array}$ & $\begin{array}{l}58.9 \\
{[49.5,67.7]}\end{array}$ & $\begin{array}{l}67.2 \\
{[78.8,53.0]}\end{array}$ & $\begin{array}{l}-0.8 \\
{[-2.9,1.5]}\end{array}$ & 0.8 \\
\hline Albania & $\begin{array}{l}43.5 \\
{[38.7,48.4]}\end{array}$ & $\begin{array}{l}70.1 \\
{[75.5,64.0]}\end{array}$ & $\begin{array}{l}-2.2 \\
{[-3.1,-1.3]}\end{array}$ & $\begin{array}{l}35.6 \\
{[25.8,46.8]}\end{array}$ & $\begin{array}{l}70.4 \\
{[81.3,56.5]}\end{array}$ & $\begin{array}{l}-2.9 \\
{[-4.6,-0.8]}\end{array}$ & $\begin{array}{l}44.8 \\
{[35.6,54.3]}\end{array}$ & $\begin{array}{l}63.7 \\
{[73.5,52.5]}\end{array}$ & $\begin{array}{l}-1.6 \\
{[-3.2,0.2]}\end{array}$ & 1.3 \\
\hline Moldova & $\begin{array}{l}39.1 \\
{[35.0,43.3]}\end{array}$ & $\begin{array}{l}33.4 \\
{[37.0,29.9]}\end{array}$ & $\begin{array}{l}0.8 \\
{[-0.3,1.9]}\end{array}$ & $\begin{array}{l}43.6 \\
{[32.3,55.5]}\end{array}$ & $\begin{array}{l}39.1 \\
{[50.4,28.8]}\end{array}$ & $\begin{array}{l}0.6 \\
{[-2.6,3.8]}\end{array}$ & $\begin{array}{l}38.6 \\
{[32.3,45.3]}\end{array}$ & $\begin{array}{l}41.8 \\
{[50.6,33.4]}\end{array}$ & $\begin{array}{l}-0.5 \\
{[-2.6,1.7]}\end{array}$ & -1.1 \\
\hline Tajikistan & $\begin{array}{l}38.4 \\
{[35.0,41.9]}\end{array}$ & $\begin{array}{l}39.1 \\
{[43.5,34.8]}\end{array}$ & $\begin{array}{l}-0.1 \\
{[-0.7,0.6]}\end{array}$ & $\begin{array}{l}32.8 \\
{[28.3,37.7]}\end{array}$ & $\begin{array}{l}42.8 \\
{[50.7,35.2]}\end{array}$ & $\begin{array}{l}-0.8 \\
{[-1.9,0.2]}\end{array}$ & $\begin{array}{l}41.7 \\
{[33.2,50.8]}\end{array}$ & $\begin{array}{l}36.6 \\
{[42.9,30.7]}\end{array}$ & $\begin{array}{l}0.4 \\
{[-0.8,1.7]}\end{array}$ & 1.3 \\
\hline Ukraine & $\begin{array}{l}34.3 \\
{[30.5,38.4]}\end{array}$ & $\begin{array}{l}58.3 \\
{[63.7,52.7]}\end{array}$ & $\begin{array}{l}-4.8 \\
{[-6.6,-2.9]}\end{array}$ & $\begin{array}{l}37.9 \\
{[30.1,46.4]}\end{array}$ & $\begin{array}{l}63.3 \\
{[75.1,49.7]}\end{array}$ & $\begin{array}{l}-5.1 \\
{[-9,-0.7]}\end{array}$ & $\begin{array}{l}26.5 \\
{[19.9,34.5]}\end{array}$ & $\begin{array}{l}52.1 \\
{[64.0,40.0]}\end{array}$ & $\begin{array}{l}-5.1 \\
{[-8.8,-1.1]}\end{array}$ & 0.0 \\
\hline Kazakhstan & $\begin{array}{l}16.7 \\
{[14.7,18.9]}\end{array}$ & $\begin{array}{l}35.8 \\
{[38.9,32.7]}\end{array}$ & $\begin{array}{l}-2.1 \\
{[-2.7,-1.5]}\end{array}$ & $\begin{array}{l}14.3 \\
{[10.4,19.3]}\end{array}$ & $\begin{array}{l}33.5 \\
{[39.2,28.3]}\end{array}$ & $\begin{array}{l}-2.1 \\
{[-3.2,-1.0]}\end{array}$ & $\begin{array}{l}14.3 \\
{[10.3,19.6]}\end{array}$ & $\begin{array}{l}34.5 \\
{[41.2,28.3]}\end{array}$ & $\begin{array}{l}-2.2 \\
{[-3.4,-1.0]}\end{array}$ & -0.1 \\
\hline $\begin{array}{l}\text { Kyrgyz } \\
\text { Republic }\end{array}$ & $\begin{array}{l}16.2 \\
{[14.0,18.6]}\end{array}$ & $\begin{array}{l}35.3 \\
{[40.8,30.0]}\end{array}$ & $\begin{array}{l}-2.7 \\
{[-3.8,-1.6]}\end{array}$ & $\begin{array}{l}13.2 \\
{[8.8,19.2]}\end{array}$ & $\begin{array}{l}32.9 \\
{[46.9,21.5]}\end{array}$ & $\begin{array}{l}-2.8 \\
{[-5.4,-0.3]}\end{array}$ & $\begin{array}{l}22.4 \\
{[17.3,28.6]}\end{array}$ & $\begin{array}{l}36.0 \\
{[45.3,27.7]}\end{array}$ & $\begin{array}{l}-1.9 \\
{[-4.0,0.1]}\end{array}$ & 0.9 \\
\hline \multicolumn{11}{|l|}{$\begin{array}{l}\text { Latin America } \\
\text { and the } \\
\text { Caribbean }\end{array}$} \\
\hline $\begin{array}{l}\text { Dominican } \\
\text { Republic }\end{array}$ & $\begin{array}{l}56.8 \\
{[52.5,61.1]}\end{array}$ & $\begin{array}{l}39.5 \\
{[42.0,37.1]}\end{array}$ & $\begin{array}{l}2.9 \\
{[1.8,4.0]}\end{array}$ & $\begin{array}{l}48.3 \\
{[42.6,54.0]}\end{array}$ & $\begin{array}{l}31.5 \\
{[35.2,28.1]}\end{array}$ & $\begin{array}{l}2.8 \\
{[1.2,4.3]}\end{array}$ & $\begin{array}{l}63.3 \\
{[53.1,72.4]}\end{array}$ & $\begin{array}{l}57.1 \\
{[64.8,49.0]}\end{array}$ & $\begin{array}{l}1.0 \\
{[-2.0,3.9]}\end{array}$ & -1.8 \\
\hline Haiti & $\begin{array}{l}52.6 \\
{[50.1,55.2]}\end{array}$ & $\begin{array}{l}57.1 \\
{[60.5,53.6]}\end{array}$ & $\begin{array}{l}-0.4 \\
{[-0.9,0.1]}\end{array}$ & $\begin{array}{l}50.5 \\
{[45.8,55.3]}\end{array}$ & $\begin{array}{l}51.0 \\
{[57.4,44.6]}\end{array}$ & $\begin{array}{l}0.0 \\
{[-1.1,1.0]}\end{array}$ & $\begin{array}{l}59.7 \\
{[54.4,64.9]}\end{array}$ & $\begin{array}{l}62.4 \\
{[69.6,54.7]}\end{array}$ & $\begin{array}{l}-0.2 \\
{[-1.4,0.9]}\end{array}$ & -0.2 \\
\hline Guyana & $\begin{array}{l}50.8 \\
{[47.0,54.6]}\end{array}$ & $\begin{array}{l}56.9 \\
{[61.3,52.5]}\end{array}$ & $\begin{array}{l}-0.8 \\
{[-1.8,0.3]}\end{array}$ & $\begin{array}{l}42.2 \\
{[36.3,48.4]}\end{array}$ & $\begin{array}{l}54.6 \\
{[61.8,47.2]}\end{array}$ & $\begin{array}{l}-1.6 \\
{[-3.2,0.1]}\end{array}$ & $\begin{array}{l}72.0 \\
{[63.5,79.1]}\end{array}$ & $\begin{array}{l}66.5 \\
{[77.1,54.0]}\end{array}$ & $\begin{array}{l}0.7 \\
{[-1.7,3.1]}\end{array}$ & 2.2 \\
\hline \multicolumn{11}{|l|}{$\begin{array}{l}\text { The Middle } \\
\text { East \& North } \\
\text { Africa }\end{array}$} \\
\hline Egypt & $\begin{array}{l}72.9 \\
{[71.2,74.5]}\end{array}$ & $\begin{array}{l}59.9 \\
{[61.5,58.4]}\end{array}$ & $\begin{array}{l}1.4 \\
{[1.1,1.8]}\end{array}$ & $\begin{array}{l}70.4 \\
{[67.3,73.4]}\end{array}$ & $\begin{array}{l}51.9 \\
{[55.0,48.7]}\end{array}$ & $\begin{array}{l}2.1 \\
{[1.4,2.7]}\end{array}$ & $\begin{array}{l}74.5 \\
{[70.8,77.9]}\end{array}$ & $\begin{array}{l}70.3 \\
{[74.3,65.9]}\end{array}$ & $\begin{array}{l}0.5 \\
{[-0.4,1.3]}\end{array}$ & -1.6 \\
\hline Yemen & $\begin{array}{l}47.3 \\
{[45.2,49.5]}\end{array}$ & $\begin{array}{l}70.4 \\
{[73.6,67.1]}\end{array}$ & $\begin{array}{l}-3.3 \\
{[-4.1,-2.5]}\end{array}$ & $\begin{array}{l}45.3 \\
{[40.9,49.9]}\end{array}$ & $\begin{array}{l}64.3 \\
{[70.8,57.1]}\end{array}$ & $\begin{array}{l}-2.7 \\
{[-4.3,-1.0]}\end{array}$ & $\begin{array}{l}48.3 \\
{[44.0,52.7]}\end{array}$ & $\begin{array}{l}70.8 \\
{[78.3,62.0]}\end{array}$ & $\begin{array}{l}-3.2 \\
{[-4.9,-1.3]}\end{array}$ & -0.5 \\
\hline Jordan & $\begin{array}{l}33.0 \\
{[30.4,35.6]}\end{array}$ & $\begin{array}{l}62.8 \\
{[65.3,60.3]}\end{array}$ & $\begin{array}{l}-3.0 \\
{[-3.5,-2.5]}\end{array}$ & $\begin{array}{l}30.2 \\
{[23.8,37.4]}\end{array}$ & $\begin{array}{l}53.2 \\
{[57.4,49.1]}\end{array}$ & $\begin{array}{l}-2.3 \\
{[-3.4,-1.2]}\end{array}$ & $\begin{array}{l}38.4 \\
{[34.3,42.6]}\end{array}$ & $\begin{array}{l}79.9 \\
{[86.0,72.1]}\end{array}$ & $\begin{array}{l}-4.2 \\
{[-5.2,-3.0]}\end{array}$ & -1.9 \\
\hline \multicolumn{11}{|l|}{ South Asia } \\
\hline Pakistan & $\begin{array}{l}80.4 \\
{[77.9,82.7]}\end{array}$ & $\begin{array}{l}74.1 \\
{[76.0,72.1]}\end{array}$ & $\begin{array}{l}0.6 \\
{[0.2,1.0]}\end{array}$ & $\begin{array}{l}81.3 \\
{[74.9,86.4]}\end{array}$ & $\begin{array}{l}77.7 \\
{[81.4,73.5]}\end{array}$ & $\begin{array}{l}0.3 \\
{[-0.6,1.2]}\end{array}$ & $\begin{array}{l}83.4 \\
{[79.3,86.9]}\end{array}$ & $\begin{array}{l}73.8 \\
{[78.5,68.5]}\end{array}$ & $\begin{array}{l}0.9 \\
{[0.1,1.7]}\end{array}$ & 0.5 \\
\hline India & $\begin{array}{l}58.5 \\
{[58.0,59.0]}\end{array}$ & $\begin{array}{l}76.9 \\
{[77.8,75.9]}\end{array}$ & $\begin{array}{l}-1.8 \\
{[-2.0,-1.7]}\end{array}$ & $\begin{array}{l}59.4 \\
{[58.4,60.4]}\end{array}$ & $\begin{array}{l}83.3 \\
{[84.8,81.6]}\end{array}$ & $\begin{array}{l}-2.4 \\
{[-2.6,-2.1]}\end{array}$ & $\begin{array}{l}57.1 \\
{[56.0,58.2]}\end{array}$ & $\begin{array}{l}69.7 \\
{[71.8,67.5]}\end{array}$ & $\begin{array}{l}-1.3 \\
{[-1.6,-0.9]}\end{array}$ & 1.1 \\
\hline Bangladesh & $\begin{array}{l}49.2 \\
{[46.5,51.9]}\end{array}$ & $\begin{array}{l}56.9 \\
{[59.8,54.1]}\end{array}$ & $\begin{array}{l}-1.1 \\
{[-1.9,-0.3]}\end{array}$ & $\begin{array}{l}45.3 \\
{[39.8,51.0]}\end{array}$ & $\begin{array}{l}56.7 \\
{[61.8,51.4]}\end{array}$ & $\begin{array}{l}-1.6 \\
{[-3.1,-0.1]}\end{array}$ & $\begin{array}{l}54.0 \\
{[49.3,58.6]}\end{array}$ & $\begin{array}{l}60.6 \\
{[65.5,55.5]}\end{array}$ & $\begin{array}{l}-0.9 \\
{[-2.3,0.4]}\end{array}$ & 0.7 \\
\hline Nepal & $\begin{array}{l}45.1 \\
{[42.0,48.1]}\end{array}$ & $\begin{array}{l}64.5 \\
{[67.7,61.1]}\end{array}$ & $\begin{array}{l}-1.9 \\
{[-2.6,-1.3]}\end{array}$ & $\begin{array}{l}36.8 \\
{[31.7,42.1]}\end{array}$ & $\begin{array}{l}69.0 \\
{[74.0,63.6]}\end{array}$ & $\begin{array}{l}-3.2 \\
{[-4.2,-2.2]}\end{array}$ & $\begin{array}{l}48.7 \\
{[42.9,54.6]}\end{array}$ & $\begin{array}{l}59.5 \\
{[66.4,52.2]}\end{array}$ & $\begin{array}{l}-1.1 \\
{[-2.4,0.2]}\end{array}$ & 2.1 \\
\hline
\end{tabular}




\begin{tabular}{|c|c|c|c|c|c|c|c|c|c|c|}
\hline Chad & $\begin{array}{l}77.0 \\
{[74.6,79.2]}\end{array}$ & $\begin{array}{l}67.5 \\
{[70.7,64.2]}\end{array}$ & $\begin{array}{l}1.0 \\
{[0.4,1.5]}\end{array}$ & $\begin{array}{l}77.9 \\
{[74.5,81.0]}\end{array}$ & $\begin{array}{l}58.6 \\
{[68.0,48.5]}\end{array}$ & $\begin{array}{l}1.9 \\
{[0.7,3.3]}\end{array}$ & $\begin{array}{l}71.8 \\
{[67.2,76.0]}\end{array}$ & $\begin{array}{l}63.9 \\
{[69.3,58.1]}\end{array}$ & $\begin{array}{l}0.8 \\
{[-0.2,1.8]}\end{array}$ & -1.1 \\
\hline Cote d'Ivoire & $\begin{array}{l}69.2 \\
{[65.7,72.5]}\end{array}$ & $\begin{array}{l}75.1 \\
{[78.0,71.9]}\end{array}$ & $\begin{array}{l}-1.2 \\
{[-2.5,0.1]}\end{array}$ & $\begin{array}{l}52.9 \\
{[62.5,75.8]}\end{array}$ & $\begin{array}{l}77.1 \\
{[81.7,71.8]}\end{array}$ & $\begin{array}{l}-4.8 \\
{[-3.8,0.8]}\end{array}$ & $\begin{array}{l}69.7 \\
{[64.1,74.8]}\end{array}$ & $\begin{array}{l}76.1 \\
{[82.8,67.8]}\end{array}$ & $\begin{array}{l}-1.3 \\
{[-3.7,1.4]}\end{array}$ & 3.6 \\
\hline Cameroon & $\begin{array}{l}68.8 \\
{[66.1,71.4]}\end{array}$ & $\begin{array}{l}80.4 \\
{[82.5,78.1]}\end{array}$ & $\begin{array}{l}-1.5 \\
{[-2.1,-0.8]}\end{array}$ & $\begin{array}{l}78.3 \\
{[73.6,82.3]}\end{array}$ & $\begin{array}{l}81.9 \\
{[85.8,77.2]}\end{array}$ & $\begin{array}{l}-0.5 \\
{[-1.5,0.6]}\end{array}$ & $\begin{array}{l}61.5 \\
{[56.6,66.1]}\end{array}$ & $\begin{array}{l}69.8 \\
{[75.3,63.7]}\end{array}$ & $\begin{array}{l}-1.0 \\
{[-2.3,0.3]}\end{array}$ & -0.6 \\
\hline Nigeria & $\begin{array}{l}66.8 \\
{[65.0,68.6]}\end{array}$ & $\begin{array}{l}69.4 \\
{[73.1,65.4]}\end{array}$ & $\begin{array}{l}-0.3 \\
{[-0.8,0.3]}\end{array}$ & $\begin{array}{l}69.9 \\
{[66.4,73.3]}\end{array}$ & $\begin{array}{l}75.5 \\
{[80.8,69.3]}\end{array}$ & $\begin{array}{l}-0.6 \\
{[-1.4,0.4]}\end{array}$ & $\begin{array}{l}62.1 \\
{[59.3,64.9]}\end{array}$ & $\begin{array}{l}69.4 \\
{[77.4,60.0]}\end{array}$ & $\begin{array}{l}-0.7 \\
{[-1.8,0.5]}\end{array}$ & -0.2 \\
\hline Senegal & $\begin{array}{l}66.4 \\
{[64.2,68.6]}\end{array}$ & $\begin{array}{l}77.4 \\
{[80.0,74.5]}\end{array}$ & $\begin{array}{l}-0.9 \\
{[-1.3,-0.5]}\end{array}$ & $\begin{array}{l}63.2 \\
{[59.0,67.1]}\end{array}$ & $\begin{array}{l}77.9 \\
{[83 \cdot 0,71.9]}\end{array}$ & $\begin{array}{l}-1.2 \\
{[-2,-0.4]}\end{array}$ & $\begin{array}{l}71.7 \\
{[67.1,75.9]}\end{array}$ & $\begin{array}{l}83.6 \\
{[87.6,78.6]}\end{array}$ & $\begin{array}{l}-1.0 \\
{[-1.7,-0.2]}\end{array}$ & 0.2 \\
\hline Thailand & $\begin{array}{l}60.1 \\
{[55.6,64.4]}\end{array}$ & $\begin{array}{l}50.4 \\
{[53.6,47.2]}\end{array}$ & $\begin{array}{l}0.9 \\
{[0.2,1.6]}\end{array}$ & $\begin{array}{l}47.0 \\
{[38.6,55.6]}\end{array}$ & $\begin{array}{l}46.2 \\
{[54.3,38.3]}\end{array}$ & $\begin{array}{l}0.1 \\
{[-1.4,1.6]}\end{array}$ & $\begin{array}{l}72.8 \\
{[61.7,81.6]}\end{array}$ & $\begin{array}{l}57.3 \\
{[63.1,51.2]}\end{array}$ & $\begin{array}{l}1.4 \\
{[-0.1,2.8]}\end{array}$ & 1.3 \\
\hline Gambia & $\begin{array}{l}48.5 \\
{[43.9,53.1]}\end{array}$ & $\begin{array}{l}52.3 \\
{[54.9,49.6]}\end{array}$ & $\begin{array}{l}-0.5 \\
{[-1.4,0.4]}\end{array}$ & $\begin{array}{l}41.5 \\
{[35.2,48.1]}\end{array}$ & $\begin{array}{l}51.6 \\
{[56.6,46.6]}\end{array}$ & $\begin{array}{l}-1.3 \\
{[-2.7,0.2]}\end{array}$ & $\begin{array}{l}51.1 \\
{[43.5,58.6]}\end{array}$ & $\begin{array}{l}52.3 \\
{[57.2,47.4]}\end{array}$ & $\begin{array}{l}-0.1 \\
{[-1.7,1.4]}\end{array}$ & 1.1 \\
\hline $\begin{array}{l}\text { Democratic } \\
\text { Republic of } \\
\text { Congo }\end{array}$ & $\begin{array}{l}48.1 \\
{[45.3,50.9]}\end{array}$ & $\begin{array}{l}52.0 \\
{[56.3,47.6]}\end{array}$ & $\begin{array}{l}-0.7 \\
{[-1.8,0.5]}\end{array}$ & $\begin{array}{l}69.5 \\
{[48.6,57.2]}\end{array}$ & $\begin{array}{l}57.1 \\
{[64.7,49.3]}\end{array}$ & $\begin{array}{l}2.1 \\
{[-2.7,1.3]}\end{array}$ & $\begin{array}{l}47.9 \\
{[41.8,54.0]}\end{array}$ & $\begin{array}{l}53.2 \\
{[59.0,47.3]}\end{array}$ & $\begin{array}{l}-0.9 \\
{[-2.9,1.1]}\end{array}$ & -3.0 \\
\hline Niger & $\begin{array}{l}47.1 \\
{[44.2,49.9]}\end{array}$ & $\begin{array}{l}53.4 \\
{[56.1,50.7]}\end{array}$ & $\begin{array}{l}-1.1 \\
{[-2 \cdot 0,-0.1]}\end{array}$ & $\begin{array}{l}48.1 \\
{[43.5,52.8]}\end{array}$ & $\begin{array}{l}59.6 \\
{[64.7,54.3]}\end{array}$ & $\begin{array}{l}-1.9 \\
{[-3.5,-0.3]}\end{array}$ & $\begin{array}{l}39.0 \\
{[34.7,43.5]}\end{array}$ & $\begin{array}{l}43.2 \\
{[48.0,38.6]}\end{array}$ & $\begin{array}{l}-0.7 \\
{[-2.2,0.8]}\end{array}$ & 1.2 \\
\hline Sierra Leone & $\begin{array}{l}46.2 \\
{[42.6,49.8]}\end{array}$ & $\begin{array}{l}66.9 \\
{[70.1,63.5]}\end{array}$ & $\begin{array}{l}-2.6 \\
{[-3.4,-1.7]}\end{array}$ & $\begin{array}{l}45.7 \\
{[39.5,52.1]}\end{array}$ & $\begin{array}{l}58.6 \\
{[65.2,51.7]}\end{array}$ & $\begin{array}{l}-1.6 \\
{[-3.2,0.0]}\end{array}$ & $\begin{array}{l}50.0 \\
{[44.6,55.4]}\end{array}$ & $\begin{array}{l}73.4 \\
{[79.1,66.7]}\end{array}$ & $\begin{array}{l}-2.9 \\
{[-4.3,-1.4]}\end{array}$ & -1.3 \\
\hline Benin & $\begin{array}{l}45.9 \\
{[43.7,48.2]}\end{array}$ & $\begin{array}{l}45.9 \\
{[48.0,43.9]}\end{array}$ & $\begin{array}{l}0.0 \\
{[-0.4,0.4]}\end{array}$ & $\begin{array}{l}46.7 \\
{[42.7,50.7]}\end{array}$ & $\begin{array}{l}50.4 \\
{[54.0,46.8]}\end{array}$ & $\begin{array}{l}-0.3 \\
{[-1.0,0.4]}\end{array}$ & $\begin{array}{l}47.4 \\
{[43.5,51.3]}\end{array}$ & $\begin{array}{l}43.4 \\
{[47.1,39.8]}\end{array}$ & $\begin{array}{l}0.4 \\
{[-0.3,1.0]}\end{array}$ & 0.7 \\
\hline Zimbabwe & $\begin{array}{l}42.4 \\
{[39.4,45.4]}\end{array}$ & $\begin{array}{l}31.8 \\
{[35.2,28.6]}\end{array}$ & $\begin{array}{l}1.1 \\
{[0.4,1.7]}\end{array}$ & $\begin{array}{l}40.4 \\
{[34.5,46.5]}\end{array}$ & $\begin{array}{l}36.6 \\
{[45.9,28.2]}\end{array}$ & $\begin{array}{l}0.4 \\
{[-1.1,1.8]}\end{array}$ & $\begin{array}{l}43.2 \\
{[37.6,49.0]}\end{array}$ & $\begin{array}{l}25.5 \\
{[33.4,19.0]}\end{array}$ & $\begin{array}{l}1.8 \\
{[0.4,3.0]}\end{array}$ & 1.4 \\
\hline Mali & $\begin{array}{l}42.2 \\
{[39.7,44.8]}\end{array}$ & $\begin{array}{l}55.6 \\
{[57.9,53.2]}\end{array}$ & $\begin{array}{l}-2.2 \\
{[-3.0,-1.4]}\end{array}$ & $\begin{array}{l}43.1 \\
{[38.7,47.5]}\end{array}$ & $\begin{array}{l}58.1 \\
{[63.0,53.0]}\end{array}$ & $\begin{array}{l}-2.5 \\
{[-4.1,-0.9]}\end{array}$ & $\begin{array}{l}43.9 \\
{[38.2,49.7]}\end{array}$ & $\begin{array}{l}43.8 \\
{[48.4,39.3]}\end{array}$ & $\begin{array}{l}0.0 \\
{[-1.7,1.7]}\end{array}$ & 2.5 \\
\hline Togo & $\begin{array}{l}39.4 \\
{[36.5,42.4]}\end{array}$ & $\begin{array}{l}64.2 \\
{[67.0,61.2]}\end{array}$ & $\begin{array}{l}-3.5 \\
{[-4.4,-2.7]}\end{array}$ & $\begin{array}{l}39.9 \\
{[35.2,44.9]}\end{array}$ & $\begin{array}{l}71.2 \\
{[75.7,66.2]}\end{array}$ & $\begin{array}{l}-4.5 \\
{[-5.8,-3.0]}\end{array}$ & $\begin{array}{l}32.3 \\
{[27.4,37.7]}\end{array}$ & $\begin{array}{l}60.0 \\
{[66.6,53.0]}\end{array}$ & $\begin{array}{l}-4.0 \\
{[-5.6,-2.2]}\end{array}$ & 0.5 \\
\hline Liberia & $\begin{array}{l}38.8 \\
{[34.9,42.7]}\end{array}$ & $\begin{array}{l}33.8 \\
{[36.9,30.9]}\end{array}$ & $\begin{array}{l}0.7 \\
{[-0.3,1.7]}\end{array}$ & $\begin{array}{l}38.7 \\
{[32.9,44.8]}\end{array}$ & $\begin{array}{l}35.5 \\
{[42.9,28.8]}\end{array}$ & $\begin{array}{l}0.5 \\
{[-1.4,2.3]}\end{array}$ & $\begin{array}{l}46.4 \\
{[39.4,53.6]}\end{array}$ & $\begin{array}{l}37.5 \\
{[45.7,29.9]}\end{array}$ & $\begin{array}{l}1.3 \\
{[-0.9,3.4]}\end{array}$ & 0.8 \\
\hline Lesotho & $\begin{array}{l}34.7 \\
{[31 \cdot 6,37.9]}\end{array}$ & $\begin{array}{l}43.2 \\
{[45.9,40.4]}\end{array}$ & $\begin{array}{l}-0.9 \\
{[-1.4,-0.3]}\end{array}$ & $\begin{array}{l}28.6 \\
{[23.0,35.0]}\end{array}$ & $\begin{array}{l}44.5 \\
{[49.8,39.3]}\end{array}$ & $\begin{array}{l}-1.6 \\
{[-2.7,-0.4]}\end{array}$ & $\begin{array}{l}40.9 \\
{[33.5,48.6]}\end{array}$ & $\begin{array}{l}42.3 \\
{[49.5,35.5]}\end{array}$ & $\begin{array}{l}-0.1 \\
{[-1.6,1.3]}\end{array}$ & 1.5 \\
\hline Zambia & $\begin{array}{l}34.2 \\
{[32.1,36.4]}\end{array}$ & $\begin{array}{l}44.4 \\
{[46.6,42.2]}\end{array}$ & $\begin{array}{l}-1.7 \\
{[-2.4,-1.0]}\end{array}$ & $\begin{array}{l}35.5 \\
{[32.1,39.1]}\end{array}$ & $\begin{array}{l}44.4 \\
{[49.0,39.9]}\end{array}$ & $\begin{array}{l}-1.5 \\
{[-2.8,-0.1]}\end{array}$ & $\begin{array}{l}33.6 \\
{[29.8,37.6]}\end{array}$ & $\begin{array}{l}45.4 \\
{[52.6,38.5]}\end{array}$ & $\begin{array}{l}-2.0 \\
{[-3.8,-0.2]}\end{array}$ & -0.5 \\
\hline Uganda & $\begin{array}{l}33.9 \\
{[32.1,35.8]}\end{array}$ & $\begin{array}{l}58.2 \\
{[61.0,55.4]}\end{array}$ & $\begin{array}{l}-2.4 \\
{[-2.9,-2.0]}\end{array}$ & $\begin{array}{l}41.3 \\
{[37.7,45.1]}\end{array}$ & $\begin{array}{l}59.3 \\
{[64.2,54.3]}\end{array}$ & $\begin{array}{l}-1.8 \\
{[-2.7,-0.9]}\end{array}$ & $\begin{array}{l}28.1 \\
{[24.3,32.3]}\end{array}$ & $\begin{array}{l}52.1 \\
{[58.6,45.6]}\end{array}$ & $\begin{array}{l}-2.4 \\
{[-3.4,-1.3]}\end{array}$ & -0.6 \\
\hline Namibia & $\begin{array}{l}28.8 \\
{[26.6,31.1]}\end{array}$ & $\begin{array}{l}32.7 \\
{[35.3,30.1]}\end{array}$ & $\begin{array}{l}-0.6 \\
{[-1.2,0.1]}\end{array}$ & $\begin{array}{l}27.7 \\
{[22.8,33.3]}\end{array}$ & $\begin{array}{l}26.4 \\
{[31.2,22.1]}\end{array}$ & $\begin{array}{l}0.2 \\
{[-1.2,1.6]}\end{array}$ & $\begin{array}{l}33.8 \\
{[28.9,39.0]}\end{array}$ & $\begin{array}{l}38.3 \\
{[47.4,29.9]}\end{array}$ & $\begin{array}{l}-0.6 \\
{[-2.6,1.3]}\end{array}$ & -0.8 \\
\hline Ethiopia & $\begin{array}{l}26.7 \\
{[24.4,29.1]}\end{array}$ & $\begin{array}{l}33.8 \\
{[36.5,31.2]}\end{array}$ & $\begin{array}{l}-0.6 \\
{[-1.1,-0.2]}\end{array}$ & $\begin{array}{l}27.7 \\
{[22.9,33.1]}\end{array}$ & $\begin{array}{l}30.1 \\
{[34.9,25.6]}\end{array}$ & $\begin{array}{l}-0.2 \\
{[-1.1,0.7]}\end{array}$ & $\begin{array}{l}23.7 \\
{[19.7,28.2]}\end{array}$ & $\begin{array}{l}38.3 \\
{[44.8,32.3]}\end{array}$ & $\begin{array}{l}-1.3 \\
{[-2 \cdot 3,-0.4]}\end{array}$ & -1.1 \\
\hline Malawi & $\begin{array}{l}23.8 \\
{[22.1,25.5]}\end{array}$ & $\begin{array}{l}32.3 \\
{[34.2,30.4]}\end{array}$ & $\begin{array}{l}-0.8 \\
{[-1.1,-0.4]}\end{array}$ & $\begin{array}{l}24.6 \\
{[21.6,27.9]}\end{array}$ & $\begin{array}{l}38.2 \\
{[42.1,34.4]}\end{array}$ & $\begin{array}{l}-1.2 \\
{[-1.9,-0.6]}\end{array}$ & $\begin{array}{l}27.6 \\
{[24.3,31.2]}\end{array}$ & $\begin{array}{l}23.4 \\
{[28.2,19.1]}\end{array}$ & $\begin{array}{l}0.4 \\
{[-0.4,1.1]}\end{array}$ & 1.6 \\
\hline
\end{tabular}

Figure 4 demonstrates the variation in the pace of change in the prevalence of delayed breastfeeding initiation between the poorest and wealthiest quintiles against the absolute change per year. The national prevalence of delayed breastfeeding initiation reduced over time in 30 countries. While the national prevalence increased in the Dominican Republic, Egypt and Chad, the increase was faster in the poorest quintile than the wealthiest quintile. At the same time, the reduction in delayed breastfeeding initiation in the poorest quintile outpaced the drop in the wealthiest quintiles in 12 countries, with the largest decline in the poorest quintile in Cote'd Ivoire. Similarly, in Cambodia, Mali, Nepal and Guyana, the prevalence of delayed breastfeeding initiation decreased, favouring the poorest quintile (Fig. 4: top left quadrant). Zimbabwe, Thailand, Liberia, and Pakistan experienced a national increase in delayed breastfeeding initiation (Fig. 4: top right quadrant), with the increase among the poorest quintile outpacing the wealthiest in these countries.

\section{Discussion}

\section{Main Findings}

The inequality in the prevalence of delayed breastfeeding initiation does not depict a clear pro-poor or pro-rich pattern. In $64 \%$ ( $n=37$ ) of our study countries, delayed breastfeeding initiation was higher among the wealthiest (pro-poor) while it was higher among the poorest in 21 countries (pro-rich). The prevalence of delayed breastfeeding initiation and the extent of within-country differences varied across wealth quintiles and countries, with the 
highest within-country difference in Thailand and Guyana. Our findings suggest that pro-breastfeeding programs must consider the socio-economic context within the country and how it relates to post-birth health service patterns. They also suggest a need to further enhance the facilitation of the postbirth care platform in these countries and make it accessible to families of all socio-economic statuses irrespective of their mode and place of childbirth.

The pro-poor inequality found in this paper suggests that the wealthiest families in many LMICs require appropriate encouragement and support to ensure proper breastfeeding practices. Governments and program managers should include evidence-based care platforms to support appropriate and timely breastfeeding practices immediately after childbirth, regardless of a woman's socio-economic status. Our results will help governments and program managers better assess the impact of the socio-economic profile of a country when designing and implementing integrated and contextually appropriate strategies to improve breastfeeding initiation practices. Findings from this study will allow health managers to learn from experiences and practices in other socioeconomically comparable countries.

Overall, we found a weak correlation between income inequality and wealth-related inequality in delayed breastfeeding initiation in the study countries. The highest level of absolute inequality (pro-poor) in the prevalence of delayed breastfeeding initiation was observed in Latin America and the Caribbean. Income inequality is historically known to be higher in this region [43]. We also found income inequality and absolute difference in delayed breastfeeding initiation higher in this region than other world regions and had the strongest correlation. The high absolute inequality in Latin American countries suggests that significant reforms are required in promoting pro-breastfeeding strategies and guidelines while accounting for the local practices, especially among those in the wealthiest quintile. However, in general, Latin American LMICs have a lower prevalence of delayed breastfeeding initiation [44]. Our results confirm that absolute inequality in breastfeeding initiation is not correlated with income inequality in the Sub-Saharan African region. Thirteen SubSaharan African countries had a pro-rich inequality, suggesting the need to implement pro-poor strategies to support appropriate breastfeeding practices while accounting for local-level dynamics and cultural practices.

\section{Strengths and limitations}

The key strength of this study was that we used large nationally representative surveys from 58 countries spanning six regions of the world. The use of comparable and standardized data collected from large nationwide population samples allowed us to present consistent comparisons of within-country inequalities in breastfeeding initiation rates over time.

Our study has some limitations. Firstly, we could not portray the within-country cultural practices and beliefs surrounding the timely initiation of breastfeeding. Such cultural beliefs could help us better understand the combined role of socio-economic status and cultural context in influencing delayed breastfeeding initiation. However, this information is not within the scope of the data collection in the DHS and MICs surveys and hence could not be explored. Secondly, this study does not include data from all low- and middle-income countries from all the world regions. Interesting observations from any of the missed countries may have further enriched our interpretations. Moreover, in this study, we included few countries from Latin America and the Caribbean, and thus, findings may not be regionally representative. Thirdly, the surveys recorded breastfeeding initiation time from mothers' responses taken at the survey time. This approach could introduce both reporting and recall bias as mothers whose infants were healthy at the time of the survey are more likely to report appropriate breastfeeding initiation than mothers whose infants experienced poor health or died before the survey. Lastly, women from higher wealth quintiles are more likely to experience caesarean section birth. They may not be aware of the actual time of breastfeeding initiation in the post-surgical period. Data from health personnel present at the time of childbirth would be more informative. However, such triangulation of information was not within the scope of the DHS and MICS surveys.

\section{Possible reasons for findings}

The reasons for the variation in delayed breastfeeding initiation within each country are complex and context-specific. The higher prevalence of caesarean section births (which increase the likelihood of delayed breastfeeding initiation) [45-47] among the wealthiest quintile [48, 49] partly explains the pro-poor inequality. Several previous studies have explored reasons for the higher likelihood of delayed breastfeeding initiation among caesarean section births [46, 47]. Mothers in the wealthiest quintile are more likely to experience caesarean section births, which increases their likelihood of delayed breastfeeding initiation [50,51]. Similarly, lower rates of caesarean section births in countries experiencing a pro-rich inequality, most of which are in Sub-Saharan Africa, can explain the higher prevalence of delayed initiation among the poorest. Studies have reported factors like mother's education, parity, skilled attendance at childbirth, home delivery, and urban residence as barriers for timely breastfeeding initiation, especially among the poorest quintile [50].

Further explanation may be related to the knowledge and skill level of the individuals present at the time of birth especially considering the place and mode of childbirth. Women from the poorest quintiles of countries with poor health infrastructure are more likely to experience childbirth at home without skilled health personnel $[52,53]$, increasing their likelihood of experiencing delayed breastfeeding initiation. This reason could explain the pattern observed in Niger, Chad, Ethiopia and Nigeria, where the rates of home births are high [54,55], and skilled attendance at birth is less than 50\% [56]. The pro-rich pattern of absolute inequality we detected in these countries helps explain this pattern.

This study explored the absolute place of residence inequality [56] in delayed breastfeeding initiation. When disaggregated by residence, the prevalence of delayed breastfeeding initiation differed greatly between urban and rural areas. Nearly half of the countries reported a higher level of inequality in urban than in rural areas, and three-fifth of these countries showed a pro-rich inequality. This finding suggests the need for pro-poor strategies to be developed specifically for the poorest in urban areas of 11 Sub-Saharan African countries (Democratic Republic of Congo, Sierra Leon, Malawi, Gambia, Sao Tome, Namibia, Rwanda, Zambia, Ghana, Liberia and Senegal). Overall, the regional level median prevalence of delayed breastfeeding initiation was higher in urban areas. 
We observed an absence of a prevailing global pattern in wealth-related inequality in the prevalence of delayed breastfeeding initiation, which was similar to previous studies [16] and global reports $[14,56]$. Programs promoting appropriate breastfeeding practices need to consider the within-country socioeconomic context, health system capacity, and standard feeding practices across the socio-economic gradient in each country. At the individual and community level, factors influencing a woman or her family's decision on the time of breastfeeding initiation is affected by socio-economic factors including place of residence [57], availability of and access to post-childbirth care information [58], prevalent postnatal practices in the community [59], education [60], and media exposure [25, 61].

We can learn important lessons from countries like Mali, Indonesia, Kazakhstan, Burundi, South Africa, Zimbabwe, Malawi and Rwanda, where the national prevalence of delayed initiation is lower than the global median, and the absolute inequality is close to zero. Studies from Rwanda and Malawi [62] show how intensive campaigns to increase awareness of appropriate breastfeeding practices and equipping the health workforce with professional skills to support breastfeeding initiation [14] can reduce inequality within a country.

Patterns concerning the 10-year trend in delayed breastfeeding initiation are not clear. The largest annual absolute reduction in delayed breastfeeding initiation was in Ukraine in both the poorest and the wealthiest. The pace of decline was identical in both wealth groups. According to the World Breastfeeding Trends Initiative (WBTi) [63], Ukraine is one of the top-ranking countries in Europe to adequately establish and monitor the implementation of breastfeeding policies and programs as recommended by the Global Strategy for Infant and Young Child Feeding [63, 64]. The annual absolute excess change had the highest positive value for Cote d'Ivoire, suggesting that the decrease in the prevalence of delayed breastfeeding initiation occurred faster among the poorest than the wealthiest. There has been a decline in the rate of delayed breastfeeding in Cote d'Ivoire since the government implemented integrated strategies to improve breastfeeding practices [65].

\section{Conclusion}

Our results suggest that delayed breastfeeding initiation is an undesirable health behaviour common among the rich compared to the poor in many countries. The WHO and BFHI guidelines outline the steps to successfully initiate breastfeeding regardless of all distal or proximal socio-economic factors $[9,66]$. However, most low- and middle-income countries do not have an effective, accountable monitoring system to track the implementation of the recommended strategies. Timely breastfeeding initiation is a critical practice that can accelerate a country's progress towards achieving the Sustainable Development Goals to improve maternal and child health and end malnutrition and poverty. The observed pro-poor inequality in this study suggests that delayed breastfeeding initiation can be largely prevented by incorporating adequate infrastructure and accountability and improved health service delivery in community and health care settings. Additional country-specific exploration is required to understand the factors associated with the pro-poor and prorich inequalities in breastfeeding initiation time. Such information would allow governments and program managers to develop appropriate and tailored strategies for disadvantaged subgroups to ensure equitable uptake of the practice of breastfeeding initiation with the first hour of birth.

\section{Abbreviations}

BFHI, Baby-friendly hospital initiative; DHS, Demographic and Health Survey; EENC, early essential newborn care; IQR, Interquartile range; LMIC, Low- and middle-income countries; MICS, Multiple Indicator Cluster Survey; UNDP, United Nations Development Programme; UNICEF, United Nations Children's Fund; WBT $i$, World Breastfeeding Trends Initiative; WHO, World Health Organization

\section{Declarations}

\section{Ethics approval and consent to participate}

This study was conducted according to the guidelines laid down in the Declaration of Helsinki and all procedures involving research study participants were approved by the Institutional Review Board (IRB) at ICF International. Written informed consent was obtained from all subjects/patients.

\section{Consent for publication}

Not applicable

\section{Availability of data and materials}

The datasets analysed during the current study are publicly available through the Demographic and Health Survey website [https://www.dhsprogram.com/data]

\section{Competing interests}

\section{All authors declare that they have no competing interests}

\section{Funding}

The primary author Shahreen Raihana received the Endeavour Postgraduate Scholarship from the Australian Government Department of Education for her PhD work.

\section{Authors' contributions}


SR designed and conducted the research, analyzed data, wrote the paper, and had primary responsibility for final content; SSP assisted in data extraction and analysis; TMH provided critical guidance on statistical analysis; MJD, TMH and AA critically reviewed the manuscript; and all authors: read and approved the final manuscript.

\section{Acknowledgement}

The authors would like to thank the measure DHS and MICS programs for granting access to the datasets from 58 countries for this analysis. The primary author [SR] would also like to thank the Australian government and the Endeavour Postgraduate Scholarship Program for funding and supporting her PhD program at The University of Sydney School of Public Health.

\section{References}

1. World Health Organization. Early initiation of breastfeeding to promote exclusive breastfeeding. 2019 https://www.who.int/elena/titles/early_breastfeeding/en/.

2. World Health Organization. Implementation guidance: protecting, promoting and supporting breastfeeding in facilities providing maternity and newborn services: the revised baby-friendly hospital initiative. 2018.

3. World Health Organization. Action plan for healthy newborn infants in the Western Pacific Region (2014-2020): Manila:. WHO Regional Office for the Western Pacific; 2014.

4. Edmond KM, Kirkwood BR, Amenga-Etego S, Owusu-Agyei S, Hurt LS. Effect of early infant feeding practices on infection-specific neonatal mortality: an investigation of the causal links with observational data from rural Ghana. Am J Clin Nutr. 2007;86(4):1126-31.

5. Clemens J, Elyazeed RA, Rao M, Savarino S, Morsy BZ, Kim Y, Wierzba T, Naficy A, Lee YJ. Early initiation of breastfeeding and the risk of infant diarrhea in rural Egypt. Pediatrics. 1999;104(1):e3.

6. Garcia C, Mullany LC, Rahmathullah L, Katz J, Thulasiraj R, Sheeladevi S, Coles C, Tielsch J. Breast-feeding initiation time and neonatal mortality risk among newborns in South India. J Perinatol. 2011;31(6):397-403.

7. Khan J, Vesel L, Bahl R, Martines JC. Timing of breastfeeding initiation and exclusivity of breastfeeding during the first month of life: effects on neonatal mortality and morbidity-a systematic review and meta-analysis. Matern Child Health J. 2015;19(3):468-79.

8. Raihana S, Dibley MJ, Rahman MM, Tahsina T, Siddique MAB, Rahman QS, Islam S, Alam A, Kelly PJ, Arifeen SE. Early initiation of breastfeeding and severe illness in the early newborn period: An observational study in rural Bangladesh. PLoS Med. 2019;16(8):e1002904.

9. Sharma IK, Byrne A. Early initiation of breastfeeding: a systematic literature review of factors and barriers in South Asia. Int Breastfeed J. $2016 ; 11: 17$.

10. Smith ER, Hurt L, Chowdhury R, Sinha B, Fawzi W, Edmond KM, Group NS. Delayed breastfeeding initiation and infant survival: A systematic review and meta-analysis. PloS one. 2017;12(7):e0180722.

11. Group NS. Timing of initiation, patterns of breastfeeding, and infant survival: prospective analysis of pooled data from three randomised trials. The Lancet Global Health. 2016;4(4):e266-75.

12. Mullany LC, Katz J, Li YM, Khatry SK, LeClerq SC, Darmstadt GL, Tielsch JM. Breast-feeding patterns, time to initiation, and mortality risk among newborns in southern Nepal. J Nutr. 2008;138(3):599-603.

13. Phukan D, Ranjan M, Dwivedi L. Impact of timing of breastfeeding initiation on neonatal mortality in India. International breastfeeding journal. 2018;13(1):1-10.

14. UNICEF and WHO. Capture the Moment - Early initiation of breastfeeding: The best start for every newborn. In. New York: UNICEF; 2018.

15. United Nations Children's Fund (UNICEF). From the first hour of life: Making the case for improved infant and young child feeding everywhere. In. https://data.unicef.org/resources/first-hour-life-new-report-breastfeeding-practices/; 2016.

16. Victora CG, Bahl R, Barros AJ, Franca GV, Horton S, Krasevec J, Murch S, Sankar MJ, Walker N, Rollins NC, et al. Breastfeeding in the 21st century: epidemiology, mechanisms, and lifelong effect. Lancet. 2016;387(10017):475-90.

17. World Health Organization (WHO). Infant and young child feeding: a tool for assessing national practices, policies and programmes. In. https://apps.who.int/iris/handle/10665/42794; 2003.

18. Whitehead M, Dahlgren G. Concepts and principles for tackling social inequities in health: Levelling up Part 1. World Health Organization: Studies on social and economic determinants of population health 2006, 2:460-474.

19. Organization WH. State of inequality: reproductive maternal newborn and child health: interactive visualization of health data. World Health Organization; 2015.

20. Adhikari M, Khanal V, Karkee R, Gavidia T. Factors associated with early initiation of breastfeeding among Nepalese mothers: further analysis of Nepal Demographic and Health Survey, 2011. International breastfeeding journal. 2014;9(1):1-9.

21. Berde AS, Yalcin SS. Determinants of early initiation of breastfeeding in Nigeria: a population-based study using the 2013 demograhic and health survey data. BMC Pregnancy Childbirth. 2016;16(1):1-9.

22. Ezeh OK, Ogbo FA, Stevens GJ, Tannous WK, Uchechukwu OL, Ghimire PR, Agho KE, Maternal G, Collaboration CHR. Factors associated with the early initiation of breastfeeding in economic community of West African States (ECOWAS). Nutrients. 2019;11(11):2765.

23. Khanal V, Scott JA, Lee AH, Karkee R, Binns CW. Factors associated with early initiation of breastfeeding in Western Nepal. Int J Environ Res Public Health. 2015;12(8):9562-74.

Page $15 / 20$ 
24. Ndirangu M, Gatimu S, Mwinyi H, Kibiwott D. Trends and factors associated with early initiation of breastfeeding in Namibia: analysis of the Demographic and Health Surveys 2000-2013. BMC Pregnancy Childbirth. 2018;18(1):1-10.

25. Seidu A-A, Ameyaw EK, Ahinkorah BO, Bonsu F. Determinants of early initiation of breastfeeding in Ghana: a population-based cross-sectional study using the 2014 Demographic and Health Survey data. BMC Pregnancy Childbirth. 2020;20(1):1-11.

26. Sharma A, Thakur PS, Tiwari R, Kasar PK, Sharma R, Kabirpanthi V. Factors associated with early initiation of breastfeeding among mothers of tribal area of Madhya Pradesh, India: a community based cross sectional study. Int J Community Med Public Heal. 2016;3(1):194-9.

27. Tilahun G, Degu G, Azale T, Tigabu A. Prevalence and associated factors of timely initiation of breastfeeding among mothers at Debre Berhan town, Ethiopia: a cross-sectional study. International breastfeeding journal. 2016;11(1):1-9.

28. UNICEF. Breastfeeding and the sustainable development goals. Factsheet. In.; 2016.

29. Micha R, Mannar V, Afshin A, Allemandi L, Baker P, Battersby J, Bhutta Z, Chen K, Corvalan C, Di Cesare M: 2020 Global nutrition report: action on equity to end malnutrition. 2020:42.

30. World Health Organization. Every Woman, Every Child, Every Adolescent: Achievements and Prospects: The Final Report of the Independent Expert Review Group on Information and Accountability for Women's and Children's Health. In.: WHO Geneva, Switzerland; 2015.

31. Croft TN, Marshall AM, Allen CK, Arnold F, Assaf S, Balian S: Guide to DHS statistics. Rockville, Maryland, USA: ICF 2018.

32. Khan S, Hancioglu A. Multiple indicator cluster surveys: delivering robust data on children and women across the globe. Stud Fam Plann. 2019;50(3):279-86.

33. Hancioglu A, Arnold F. Measuring coverage in $\mathrm{MNCH}$ : tracking progress in health for women and children using DHS and MICS household surveys. PLoS Med. 2013;10(5):e1001391.

34. World Bank. World Bank List of Economies (June 2018). In. The World Bank Group Washington, DC; 2018.

35. Rutstein SO. The DHS wealth index: approaches for rural and urban areas. Macro International Incorporated; 2008.

36. Rutstein SO. Steps to constructing the new DHS Wealth Index. Rockville: ICF International; 2015.

37. Filmer D, Pritchett LH. Estimating wealth effects without expenditure data-or tears: an application to educational enrollments in states of India. Demography. 2001;38(1):115-32.

38. Filmer D, Scott K. Assessing asset indices. Demography. 2012;49(1):359-92.

39. McKenzie DJ. Measuring inequality with asset indicators. Journal of population economics. 2005;18(2):229-60.

40. Szwarcwald CL. On the World Health Organisation's measurement of health inequalities. Journal of Epidemiology Community Health. 2002;56(3):177-82.

41. Gini index (World Bank estimate) https://data.worldbank.org/indicator/SI.POV.GINI.

42. Elkasabi M, Ren R, Pullum TW: Multilevel modeling using DHS Surveys: a framework to approximate level-weights. In: 2020 : ICF; 2020.

43. Income distribution inequality based on Gini coefficient in Latin. America as of 2017, by country [https://www.statista.com/statistics/980285/incomedistribution-gini-coefficient-latin-america-caribbean-country/].

44. Oakley L, Benova L, Macleod D, Lynch CA, Campbell OM. Early breastfeeding practices: descriptive analysis of recent demographic and health surveys. Matern Child Nutr. 2018;14(2):e12535.

45. Raihana S, Alam A, Chad N, Huda TM, Dibley MJ. Delayed Initiation of Breastfeeding and Role of Mode and Place of Childbirth: Evidence from Health Surveys in 58 Low-and Middle-Income Countries (2012-2017). International Journal of Environmental Research Public Health. 2021;18(11):5976.

46. Rowe-Murray HJ, Fisher JR. Baby friendly hospital practices: cesarean section is a persistent barrier to early initiation of breastfeeding. Birth. 2002;29(2):124-31.

47. Takahashi K, Ganchimeg T, Ota E, Vogel JP, Souza JP, Laopaiboon M, Castro CP, Jayaratne K, Ortiz-Panozo E, Lumbiganon P. Prevalence of early initiation of breastfeeding and determinants of delayed initiation of breastfeeding: secondary analysis of the WHO Global Survey. Scientific reports. 2017;7(1):1-10.

48. Boerma T, Ronsmans C, Melesse DY, Barros AJ, Barros FC, Juan L, Moller A-B, Say L, Hosseinpoor AR, Yi M. Global epidemiology of use of and disparities in caesarean sections. The Lancet. 2018;392(10155):1341-8.

49. Hasan MM, Ahmed S, Soares Magalhaes RJ, Begum T, Fatima Y, Mamun AA. Temporal trends in between and within-country inequalities in caesarean delivery in low-and middle-income countries: a Bayesian analysis. BJOG: An International Journal of Obstetrics \& Gynaecology 2021.

50. Teshale AB, Tesema GA. Timely initiation of breastfeeding and associated factors among mothers having children less than two years of age in subSaharan Africa: A multilevel analysis using recent Demographic and Health Surveys data. PloS one. 2021;16(3):e0248976.

51. Yisma E, Mol BW, Lynch JW, Smithers LG. Impact of caesarean section on breastfeeding indicators: within-country and meta-analyses of nationally representative data from 33 countries in sub-Saharan Africa. BMJ open. 2019;9(9):e027497.

52. Alam N, Hajizadeh M, Dumont A, Fournier P. Inequalities in maternal health care utilization in sub-Saharan African countries: a multiyear and multicountry analysis. PloS one. 2015;10(4):e0120922.

53. Kitaw D. Breast feeding initiation time and its impact on diarrheal disease and pneumonia in West Africa. Journal of Public Health Epidemiology. 2015;7(12):352-9.

54. Adde KS, Dickson KS, Amu H. Prevalence and determinants of the place of delivery among reproductive age women in sub-Saharan Africa. Plos one. 2020;15(12):e0244875.

Page 16/20 
55. Doctor HV, Nkhana-Salimu S, Abdulsalam-Anibilowo M. Health facility delivery in sub-Saharan Africa: successes, challenges, and implications for the 2030 development agenda. BMC Public Health. 2018;18(1):1-12.

56. Unicef. The state of the world's children 2019: statistical tables. UNICEF: New York, NY, USA; 2017.

57. Senanayake P, O'Connor E, Ogbo FA. National and rural-urban prevalence and determinants of early initiation of breastfeeding in India. BMC Public Health. 2019;19(1):1-13.

58. Rubagumya D, Abeid M, Aghan E, Noorani M: Barriers to early initiation of breastfeeding in healthy neonates in an urban hospital setting. medRxiv 2021.

59. Degefie T, Amare Y, Mulligan B. Local understandings of care during delivery and postnatal period to inform home based package of newborn care interventions in rural Ethiopia: a qualitative study. BMC International Health Human Rights. 2014;14(1):1-6.

60. Neves PA, Barros AJ, Gatica-Domínguez G, Vaz JS, Baker P, Lutter CK. Maternal education and equity in breastfeeding: trends and patterns in 81 lowand middle-income countries between 2000 and 2019. Int J Equity Health. 2021;20(1):1-13.

61. Menon P, Nguyen PH, Saha KK, Khaled A, Kennedy A, Tran LM, Sanghvi T, Hajeebhoy N, Baker J, Alayon S. Impacts on breastfeeding practices of atscale strategies that combine intensive interpersonal counseling, mass media, and community mobilization: results of cluster-randomized program evaluations in Bangladesh and Viet Nam. PLoS Med. 2016;13(10):e1002159.

62. Balogun OO, O'Sullivan EJ, McFadden A, Ota E, Gavine A, Garner CD, Renfrew MJ, MacGillivray S. Interventions for promoting the initiation of breastfeeding. Cochrane Database of Systematic Reviews 2016(11).

63. Zakarija-Grković I, Cattaneo A, Bettinelli ME, Pilato C, Vassallo C, Borg Buontempo M, Gray H, Meynell C, Wise P, Harutyunyan S. Are our babies off to a healthy start? The state of implementation of the Global strategy for infant and young child feeding in Europe. International Breastfeeding Journal. 2020;15:1-12.

64. World Health Organization. Global strategy for infant and young child feeding. World Health Organization; 2003.

65. Micondo K, Oyenusi E, Folquet A, Dainguy M, Kouadio E, Kouakou C. Breastfeeding practices and determinants in infants from birth to six months in a district of Abidjan-Cote d'Ivoire. Nigerian Journal of Paediatrics. 2016;43(3):180-5.

66. Esteves TMB, Daumas RP, Oliveira MICd, Andrade CAdFd, Leite IC. Factors associated to breastfeeding in the first hour of life: systematic review. Revista de saude publica. 2014;48:697-708.

\section{Figures}
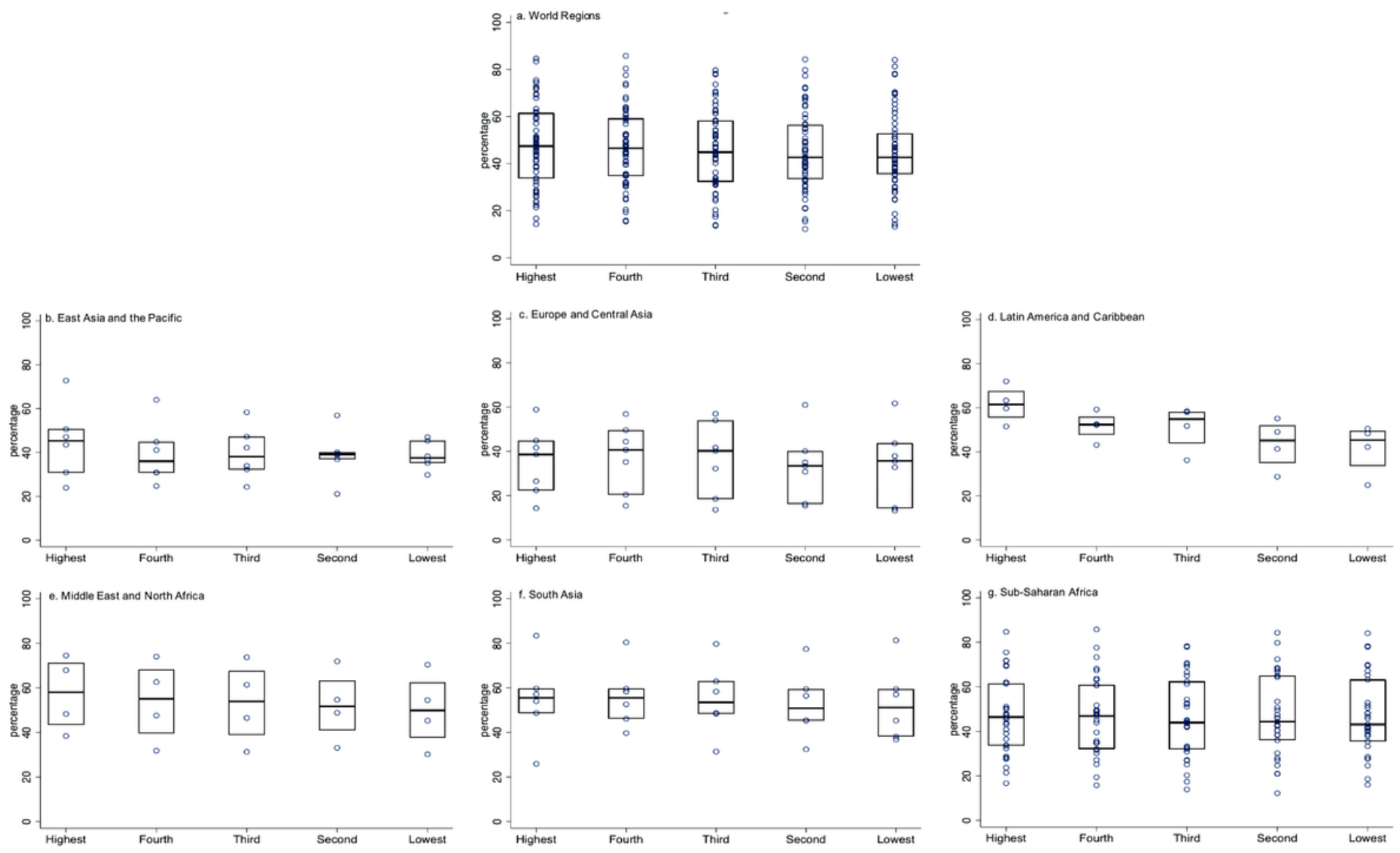

Figure 1 
Delayed breastfeeding initiation rates by economic status by World Bank regions (2012-17). Circles indicate countries, with each country represented by five circles (one for each wealth quintile). Horizontal lines indicate the median (middle point of estimates) of all countries within each subgroup, and the rectangular boxes indicate the interquartile range

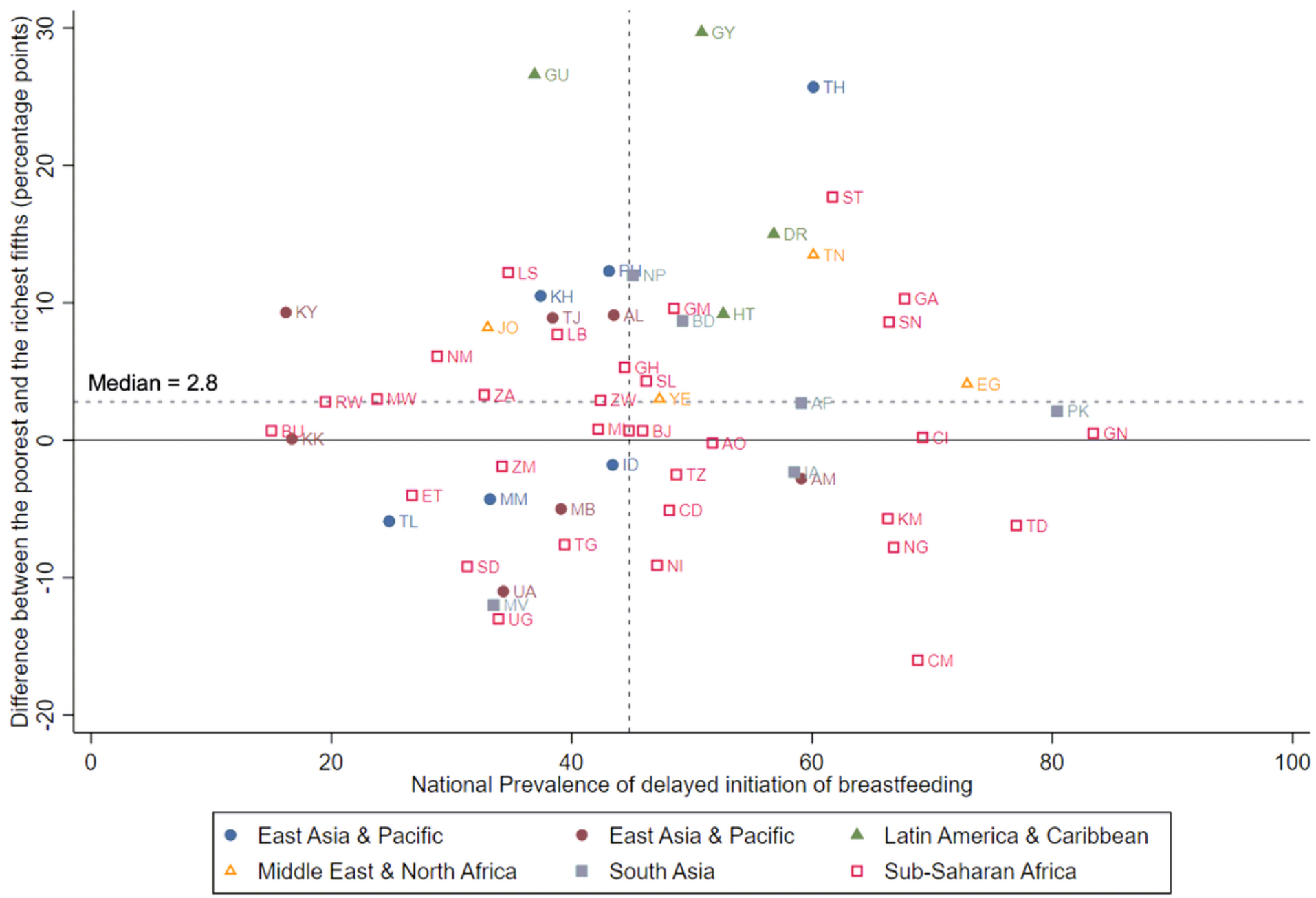

\section{Figure 2}

Delayed breastfeeding initiation rates compared with wealth-related inequality in breastfeeding initiation time (difference between wealthiest and poorest fifths) in 58 low- and middle-income countries, by World Bank region (2012-17). Shapes indicate countries, with countries in each region represented by one shape. Dashed black lines indicate the median (middle point) of all countries 


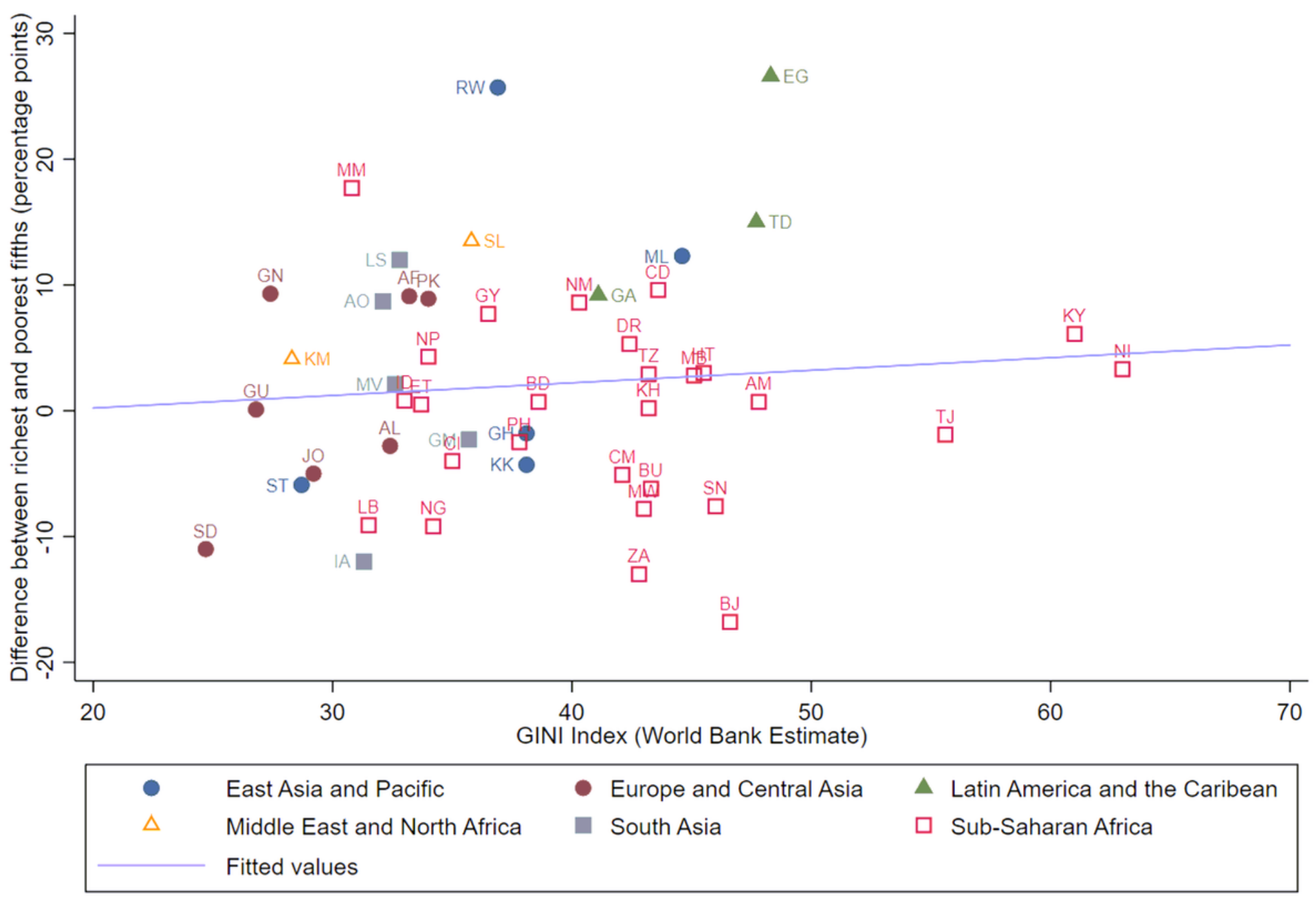

\section{Figure 3}

Income inequality (GINI index) compared with wealth-related inequality in delayed breastfeeding initiation rates (difference between wealthiest and poorest fifths) in 49 low- and middle-income countries, by World Bank region. Shapes indicate countries, with countries in each region represented by one shape. The line (best fit) indicates the association between income inequality and wealth-related inequality in delayed breastfeeding initiation 


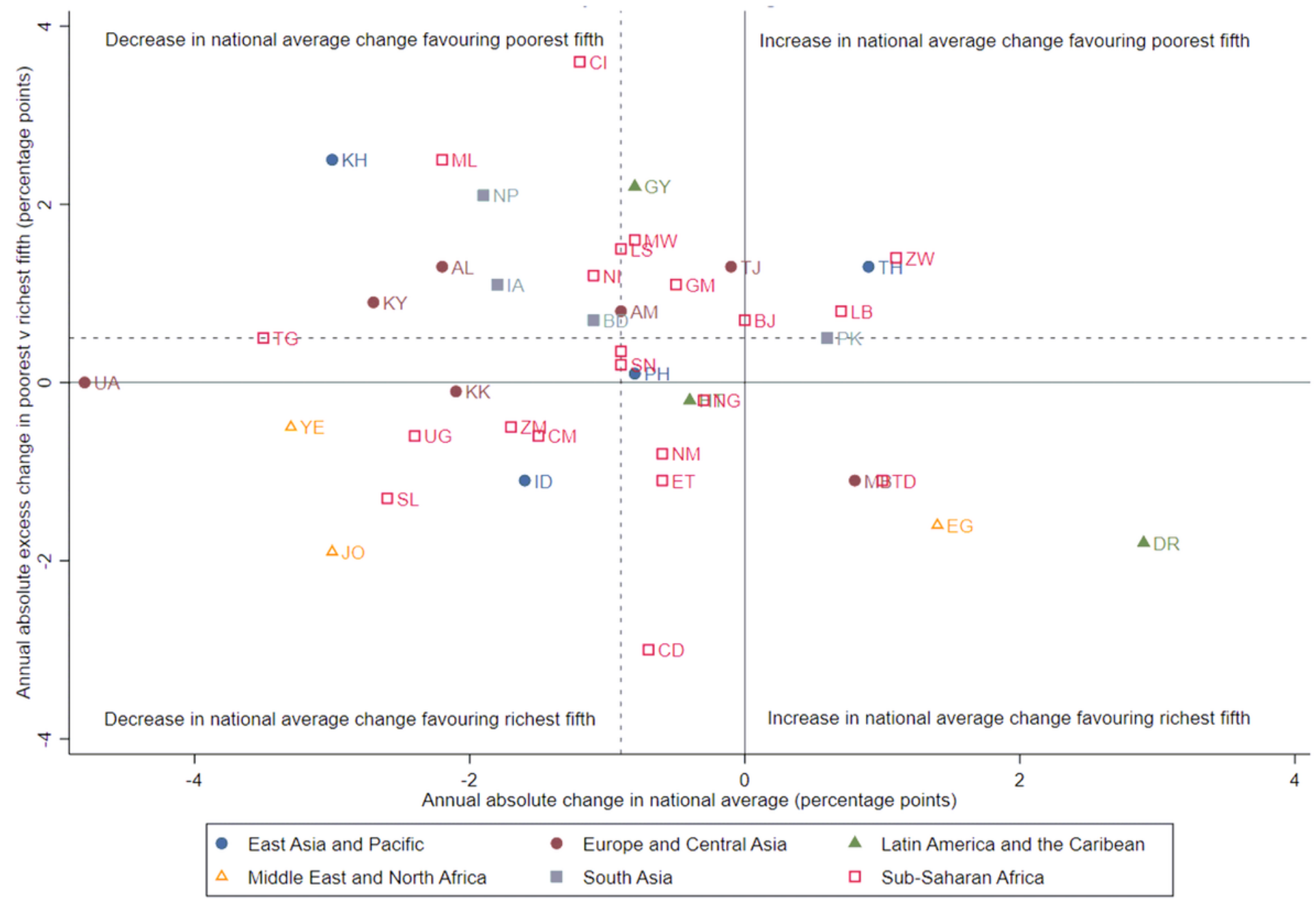

Figure 4

Change over time in national delayed breastfeeding initiation rates compared with change over time in poorest fifth compared with wealthiest fifth in 41 low- and middle-income countries, by World Bank region (2002-07 and 2012-17). Shapes indicate countries, with countries in each region represented by one shape. Dashed black lines indicate the median (middle point) of all countries

\section{Supplementary Files}

This is a list of supplementary files associated with this preprint. Click to download.

- AdditionalFile1.docx 